\title{
Shrimps of the genus Periclimenes (Crustacea, Decapoda, Palaemonidae) associated with mushroom corals (Scleractinia, Fungiidae): linking DNA barcodes to morphology
}

\author{
Cessa Rauch \\ Department of Taxonomy \& Systematics, Naturalis Biodiversity Center, P.O. Box 9517, 2300 RA \\ Leiden, The Netherlands \\ Department of Natural History, Section of Taxonomy and Evolution, University Museum \\ of Bergen, University of Bergen, $\mathrm{PB} 7800,5020$ Bergen, Norway
}

Bert W. Hoeksema

Department of Taxonomy \& Systematics, Naturalis Biodiversity Center, P.O. Box 9517, 2300 RA Leiden, The Netherlands

Bambang Hermanto

Technical Implementation Unit for Marine Biota Conservation, Research Centre for Oceanography (RCO-LIPI), Bitung, Indonesia

\section{Charles H.J.M. Fransen}

Department of Taxonomy \& Systematics, Naturalis Biodiversity Center, P.O. Box 9517, 2300 RA

Leiden, The Netherlands

charles.fransen@naturalis.nl

\begin{abstract}
Most marine palaemonid shrimp species live in symbiosis with invertebrates of various phyla. These associations range from weak epibiosis to obligatory endosymbiosis and from restricted commensalism to semi-parasitism. On coral reefs, such symbiotic shrimps can contribute to the associated biodiversity of reef corals. Among the host taxa, mushroom corals (Cnidaria: Anthozoa: Fungiidae) are known to harbour various groups of symbionts, including shrimps. Some but not all of these associated species are host-specific. Because data on the host specificity of shrimps on mushroom corals are scarce, shrimp species of the genus Periclimenes were collected from mushroom corals during fieldwork in Lembeh Strait,
\end{abstract}


North Sulawesi, Indonesia. Using molecular (COI barcoding gene) and morphological methods, three species of Periclimenes were identified: P. diversipes, $P$. watamuae and a species new to science, $P$. subcorallum sp. nov., described herein. Their host specificity was variable, with eight, three and two fungiid host records, respectively. It is concluded that shrimp species of the genus Periclimenes show much overlap in their host choice and that particular morphological traits in the host species appear to play a more important role than phylogenetic affinities within the host group.

\section{Keywords}

Caridea - Fungiidae - host corals - Indo-Pacific - new species - North Sulawesi - Scleractinia taxonomy

Zoobank: http://zoobank.org/urn:lsid:zoobank.org:pub: C34DC191-0357-4D14-BB42-D69F29E5657B

\section{Introduction}

The large majority of marine palaemonid shrimp species live in symbiosis with invertebrates of various phyla. These associations range from weak epibiosis to obligatory endosymbiosis and from restricted commensalism to semi-parasitism, with the specialisation to particular hosts likely playing a role in the diversification of this shrimp group (Horká et al., 2016). These associated shrimps species contribute to the biodiversity of coral reefs although they cannot always be found very easily (Hoeksema, 2017).

Scleractinian corals are well known as possible host species for symbiotic shrimps of the family Palaemonidae, both in the Caribbean and the Indo-Pacific. Examples are found among shrimp species of the genera Anapontonia Bruce, 1966, Ancylocaris Schenkel, 1902, Ancylomenes Okuno \& Bruce, 2010, Coralliocaris Stimpson, 186o, Ctenopontonia Bruce, 1979, Cuapetes Clark, 1919, Fennera Holthuis, 1951, Hamodactylus Holthuis, 1952, Hamopontonia Bruce, 1970, Harpiliopsis Borradaile, 1917, Harpilius Dana, 1852, Ischnopontonia Bruce, 1966, Izucaris Okuno, 1999, Jocaste Holthuis, 1952, Metapontonia Bruce, 1967, Paratypton Balss, 1914, Periclimenes O.G. Costa, 1844, Philarius
Holthuis, 1952, Platycaris Holthuis, 1952, Pliopontonia Bruce, 1973a, Pontonides Borradaile, 1917 Tectopontonia Bruce, 1973b, Vir Holthuis, 1952, and Yemenicaris Bruce, 1997 (Fransen, 1989, 1997; Fransen \& Holthuis, 2007; Okuno, 2009; De Grave \& Fransen, 2011; Fransen \& Rauch, 2013; Brinkmann \& Fransen, 2016; Horká et al., 2016; Ďuriš \& Lin, 2017). Among the scleractinian hosts, mushroom corals (Scleractinia: Fungiidae) are also known to harbour various groups of symbionts, including shrimps (De Grave, 1998; Hoeksema \& Fransen, 2011; Hoeksema et al., 2012; Fransen \& Rauch, 2013). Four of these shrimp species belong to the genus Periclimenes Costa, 1844 (Palaemonidae):P. diversipes Kemp, 1922; P.gonioporae Bruce, 1989; P. jugalis Holthuis, 1952; and P. watamuae Bruce, 1976a (Hoeksema et al., 2012), which are all members of the $P$. diversipes species group designated by Bruce (1989). This group also includes some species observed in association with other stony corals (P. difficilis Bruce, 1976b; P. madreporae Bruce, 1969; P. mahei Bruce, 1969), soft corals (P. kempi Bruce, 1969), or sponges (P. poriphilus Bruce, 2010), although $P$. difficilis probably does not belong here as it has a linguiform median plate on the $4^{\text {th }}$ thoracic sternite, which is lacking in other members of the group (Bruce, 2010). 
Data on the host specificity of Periclimenes symbionts with regards to mushroom corals are scarce (Hoeksema et al., 2012).Periclimenes diversipes has been recorded from Ctenactis crassa (Dana, 1846) (Hoeksema et al., 2012) and Herpolitha limax (Esper, 1797) (Bruce \& Coombes, 1995). This species however, has also been recorded as a symbiont of a wide range of other scleractinian corals (Fransen, 1997). Periclimenes gonioporae has been recorded from the fungiids Ctenactis echinata (Pallas, 1766), Fungia fungites (Linnaeus, 1758), Lithophyllon repanda (Dana, 1846), and Sandalolitha robusta (Quelch, 1886) (Hoeksema et al., 2012), but is also known from other scleractinians (Fransen, 1997). Periclimenes jugalis is a very rare species recorded from the mushroom coral Heliofungia actiniformis (Quoy \& Gaimard, 1833) (Hoeksema et al., 2012) but is otherwise only known from alcyonacean octocorals (Fransen, 1997). Periclimenes watamuae has been found in association with the fungiids Halomitra pileus (Linnaeus, 1758), Heliofungia actiniformis, Herpolitha limax, and Polyphyllia talpina (Lamarck, 1801) (Hoeksema et al., 2012) and is further known as a symbiont of other Scleractinia, and also of Alcyonacea (De Grave, 200o).

A faunal survey for shallow-water palaemonid shrimps associated with mushroom corals (Scleractinia: Fungiidae) during fieldwork near Lembeh Island, NE Sulawesi, Indonesia revealed three species of Periclimenes: $P$. diversipes, $P$. watamuae and a species new to science, $P$. subcorallum sp. nov., described herein (authored by Fransen \& Rauch). Most specimens were found on the underside of free-living mushroom corals, including several that constitute new host records. As the name, Periclimenes diversipes, indicates, the chelipeds of this species are morphologically diverse, which is in part related to sex and size. To a lesser extend this also counts for $P$. watamuae. Distinguishing between especially small specimens of these two species can be difficult because of their variability in cheliped shape. By sequencing cytochrome c oxidase I (COI) of multiple specimens with different sex and size from both species we aim to define several morphological and colour characters on which the two species can be distinguished.

Finally, we want to examine whether the Periclimenes species of the present study and congeneric species recorded from mushroom corals in previous studies show a hostspecific relation, and whether this relation reflects the host group phylogeny or particular morphological traits in the host group, such as attached vs. free-living mode of life (Hoeksema, 1989; Gittenberger et al., 2011; Benzoni et al., 2012) and the maximum recorded corallum size (Hoeksema, 1991; Gittenberger et al., 2011).

\section{2}

\section{Material and methods}

\subsection{Sample collection}

Specimens were collected with the help of SCUBA during a Marine Biodiversity Workshop organized in February 2012 at the Bitung field station of the Research Centre of Oceanography (PPO-LIPI). Each shrimp was photographed and then captured in a plastic bag together with its host coral and taken to the lab for examination. After identification by the second author, the host corals were returned to the field. Shrimp specimens were photographed and preserved in 95\% ethanol and their postorbital carapace length (pocl.) was measured. Data for all specimens studied are listed (table 1). Tissue samples from eggs or pleopods, were preserved in $95 \%$ ethanol before DNA extraction. Specimens are stored in the Crustacea collection of Naturalis Biodiversity Center (RMNH.CRUS.) Leiden, the Netherlands (formerly known as Rijksmuseum van Natuurlijke Historie) and the Museum Zoologicum Bogoriense, Research Center um Zoologicum Bogoriense, Research Center 


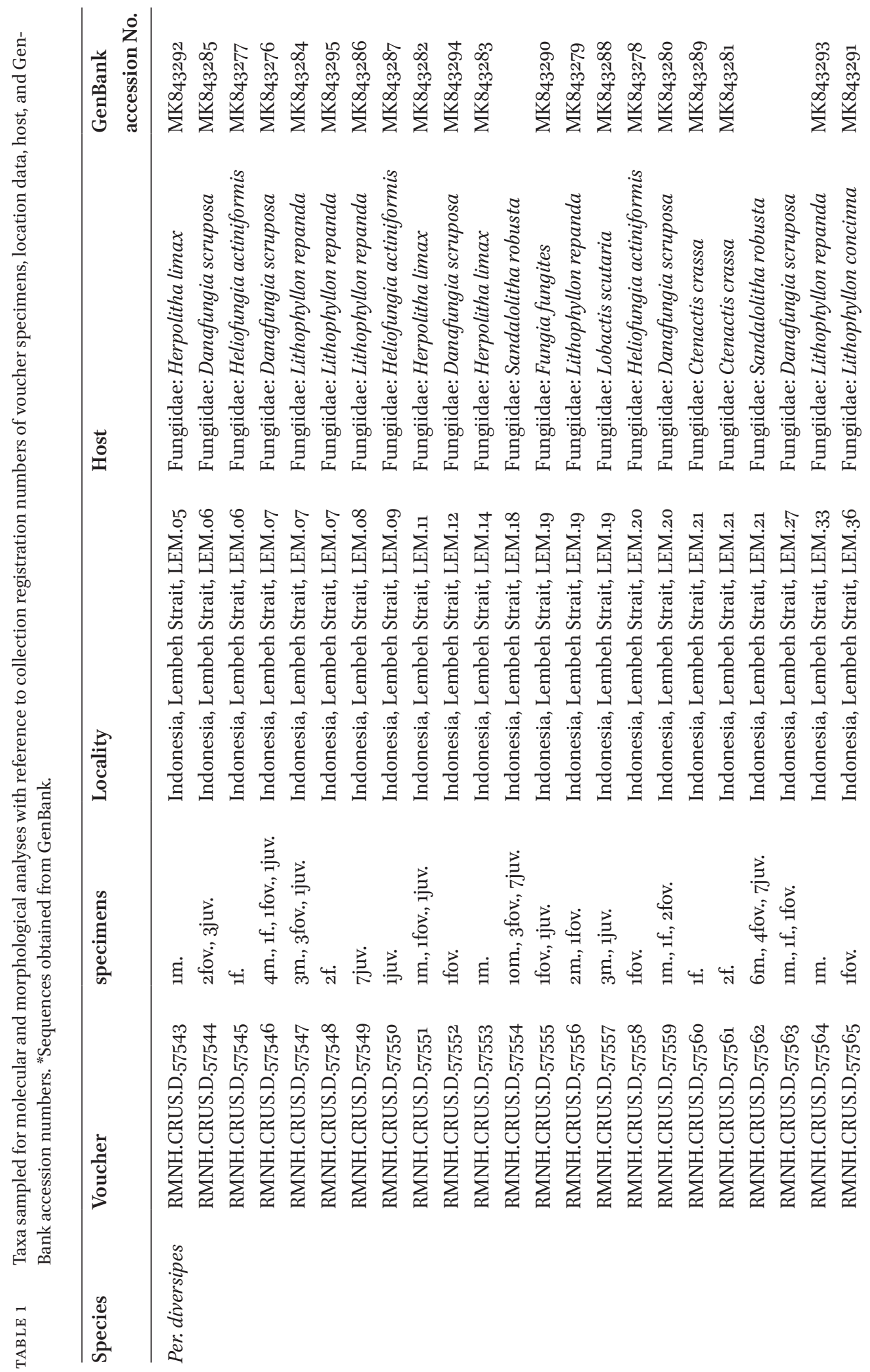



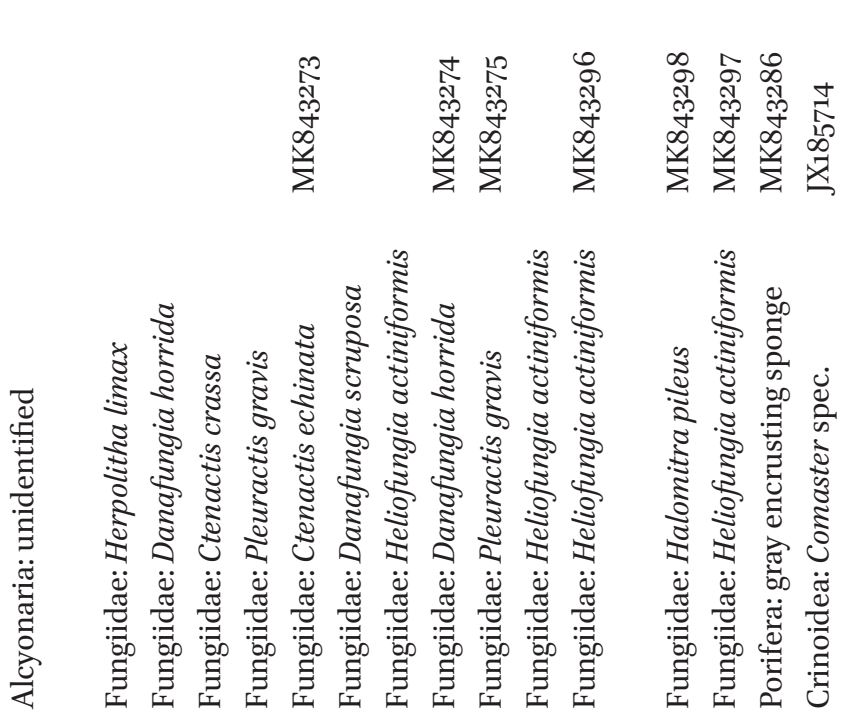

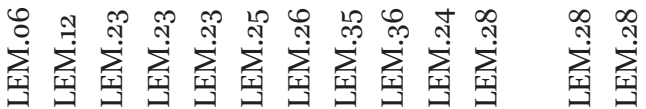

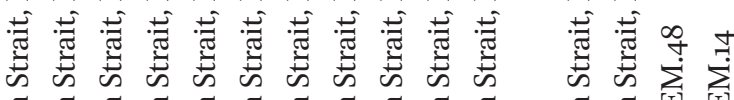

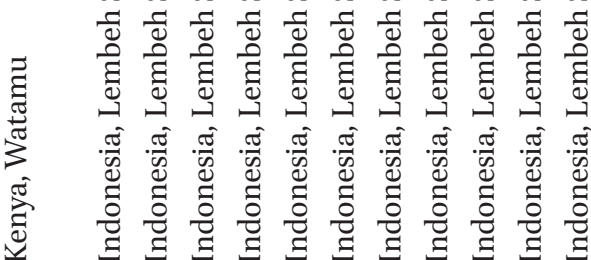

त्व

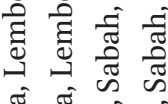

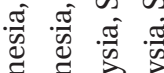

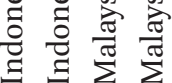

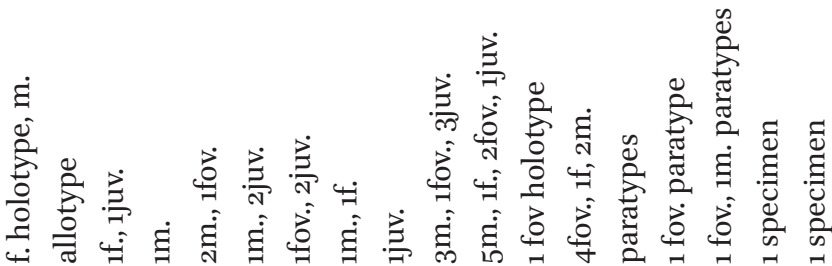

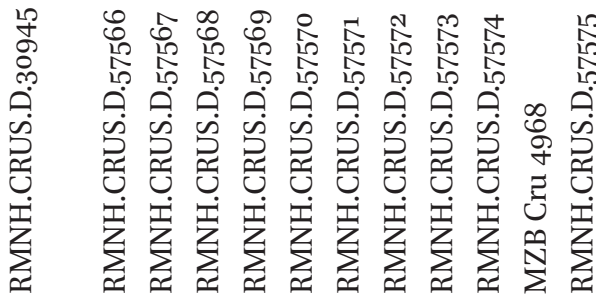

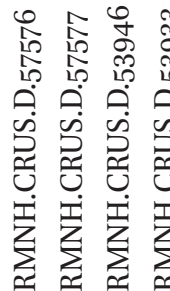

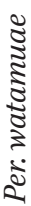

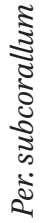

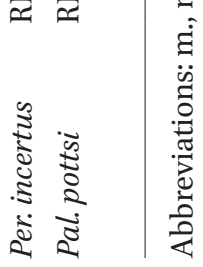


for Biology, Indonesian Institute of Sciences, Cibenong, Indonesia (MZB).

\subsection{Molecular analyses}

Total genomic DNA was extracted from eggs or pleopods using the DNeasy Blood \& Tissue Kit (QIAGEN, Hilden, Germany). Incubation lasted overnight for approximately 16 hours. The volume in the elution step was decreased to $120 \mu \mathrm{L}$ to increase the final DNA concentration. For amplifying mitochondrial cytochrome c oxidase I (COI) sequences with a polymerase chain reaction (PCR), the universal primers LCO149o and $\mathrm{HCO}_{219} 8$ (Folmer et al., 1994) were used: 5'-GGTCAACAAATCATAAAGATATTGG-3' and 5'TAAACTTCAGGGTGACCAAAAAATCA-3'. The PCR conditions were as follows: $3 \mathrm{~min}$. at $95^{\circ} \mathrm{C}$ for initial denaturing, followed by 39 cycles of $15 \mathrm{sec}$. at $95^{\circ} \mathrm{C}, 30$ sec. at $49^{\circ} \mathrm{C}, 40 \mathrm{sec}$. at $72^{\circ} \mathrm{C}$ with a final extension for $5 \mathrm{~min}$. at $72^{\circ} \mathrm{C}$. Each PCR consisted of 2.5 $\mu \mathrm{L}$ CoralLoad PCR buffer (10x; containing $15 \mathrm{mM} \mathrm{MgCl} 2$ ) (QIAGEN), $0.5 \mu \mathrm{L}$ dNTP's $(2.5 \mathrm{mM})$, 1.0 $\mu \mathrm{L}$ of each primer, $0.3 \mu \mathrm{L}$ Taq DNA polymerase ( 5 units/ $\mu \mathrm{L})$ (QIAGEN). PCR reactions were performed in volumes of $25 \mu \mathrm{L}$. Sequences were generated on an Automatic Sequencer 373oxl at Macrogen, Amsterdam. The obtained sequences were edited in Sequencher (vers. 4.10.1) and aligned with the aid of ClustalW Multiple alignment (vers. 1.4, Thompson et al., 1994) incorporated in Bioedit (vers. 5.09, Hall, 2001). Of 602 total aligned sites, 252 were variable and informative for maximum parsimony (MP). Sequences were deposited in GenBank, for which accession numbers were obtained (table 1).

\subsection{Data analyses}

Palaemonella pottsi (Borradaile, 1915) was selected as outgroup. The best-fitting model for sequence evolution $(\mathrm{GTR}+\mathrm{G})$ of the COI dataset was determined by jModelTest (vers. o.1.1., Posada, 2008), selected by the AIC (Akaike Information Criterion), and was subsequently applied to the maximum likelihood (ML) analyses with PAUP (vers. 4.ob10, Swofford, 2003) with 100 bootstrap reiterations. A maximum parsimony (MP) tree was constructed using PAUP with 2000 bootstrap reiterations of a simple heuristic search, TBR (tree bisectionreconnection) branch-swapping, and 10 randomly added sequence replications. Transversions were weighted two times over transitions.

\subsection{Morphological analyses}

In situ underwater photographs of living shrimps on their host were made with a Nikon D5 camera in a Sea \& Sea housing. Drawings were made using a Zeiss Discovery V8 and an Olympus $\mathrm{BX}_{53}$ stereo microscope, both with a drawing tube. Drawings were mounted using Adobe Photoshop CS6 software.

\subsection{Host coral morphology and phylogeny}

In order to clarify the ecological and evolutionary relation between the Periclimenes species and their host corals, their association records are projected on a cladogram of the Fungiidae based on published phylogenetic analyses (Gittenberger et al., 2011; Benzoni et al., 2012). In addition, the morphological traits attached vs. free-living mode of life and maximum recorded corallum size are projected on a similar mushroom coral cladogram based on published data (Hoeksema, 1991, 2012, 2014; Gittenberger et al., 2011).

\section{$3 \quad$ Results}

\subsection{Molecular analyses}

Both maximum likelihood and maximum parsimony analyses of COI sequences revealed three well supported clades representing the species $P$. diversipes, $P$. watamuae and an undescribed Periclimenes species (fig. 1). Haplotypes of $P$. diversipes specimens are within a range of 0.034 genetic divergence 


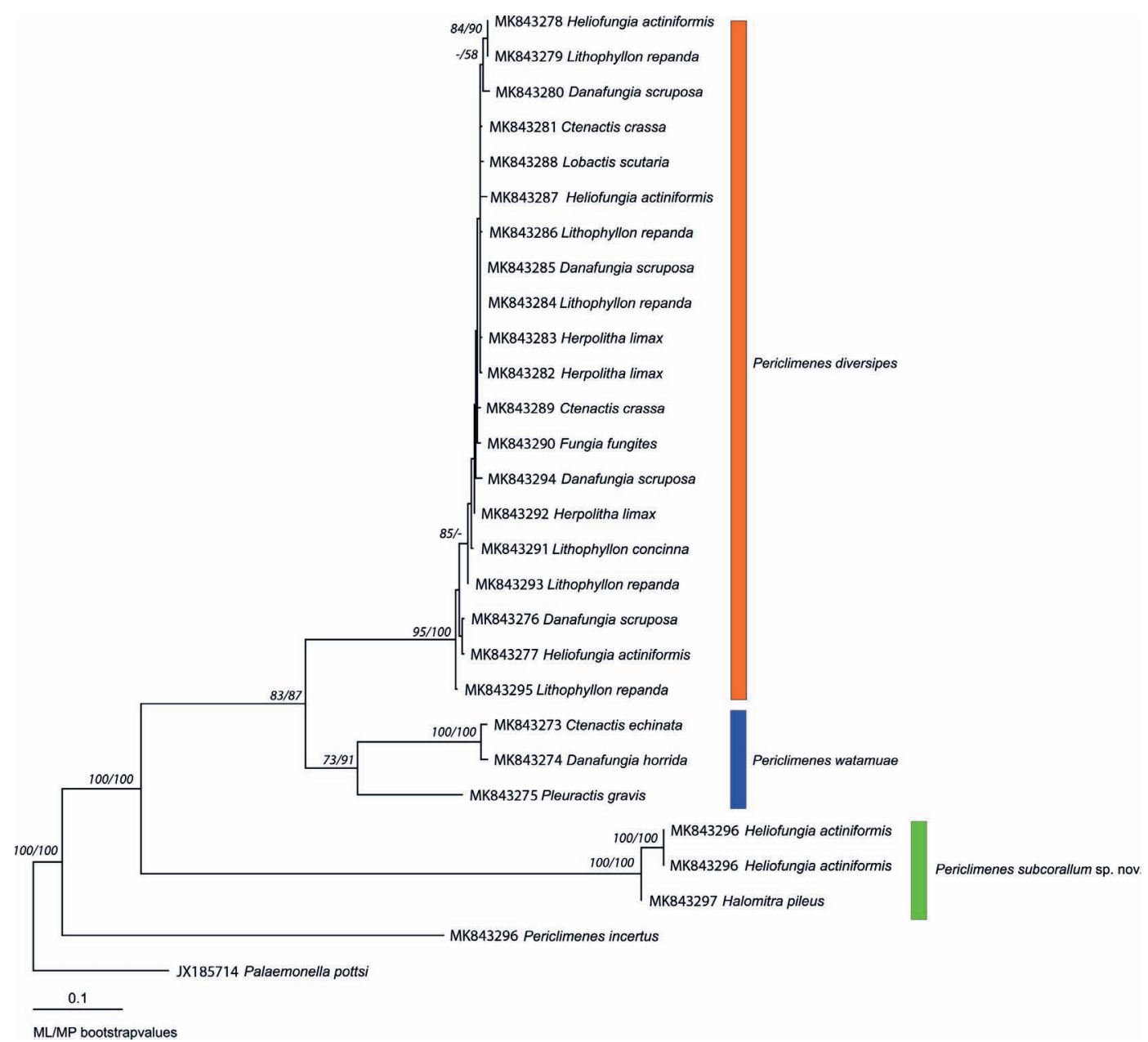

FIGURE 1 Maximum-likelihood tree using the GTR $+G$ substitution model based on cor sequence data of shrimp on mushroom coral species; GenBank accession numbers and host species indicated at end of branches; bootstrap values $<50 \%$ are not shown; bootstrap values are shown in the order ML/MP.

(table 2). There is no genetic structuring with regards to the different hosts in $P$. diversipes. The genetic distance between $P$. watamuae specimens from Ctenactis echinata and Danafungia horrida is 0.013. Between these two and the one from Pleuractis gravis is 0.187 . Morphological differences between specimens from these three hosts were not found. The genetic distance between Periclimenes subcorallum sp. nov. specimens on Heliofungia actiniformis and Halomitra pileus is $\mathbf{0 . 0 2 2}$.
Periclimenes diversipes was found associated with eight different species of mushroom coral, $P$. watamuae on three, and the new species on two species of mushroom coral (table 3).

\subsection{Morphological analyses}

Distinguishing morphological features between $P$. diversipes and $P$. watamuae were determined on the basis of specimens used in the molecular analyses; 1 ) the carpus of the tha $_{1 \text { A }}$ 
TABLE 2 JC model (Jukes \& Cantor, 1969) divergence matrix for the mitochondrial COI gene of the Periclimenes species.

\begin{tabular}{|c|c|c|c|c|c|c|c|c|c|c|c|c|c|}
\hline & Species & GenBank \# & & 2 & 3 & 4 & 5 & 6 & 7 & 8 & 9 & 10 & 11 \\
\hline 1 & Per. & MK843273 & - & & & & & & & & & & \\
\hline 2 & watamuae & MK843274 & 0.013 & - & & & & & & & & & \\
\hline 3 & & MK843275 & 0.187 & 0.187 & - & & & & & & & & \\
\hline 4 & Per. & MK843276 & 0.227 & 0.230 & 0.223 & - & & & & & & & \\
\hline 5 & diversipes & MK843277 & 0.233 & 0.236 & 0.229 & 0.003 & - & & & & & & \\
\hline 6 & & MK843278 & 0.253 & $0.25^{2}$ & 0.231 & 0.029 & 0.031 & - & & & & & \\
\hline 7 & & MK843279 & $0.25^{1}$ & $0.25^{\circ}$ & 0.229 & 0.026 & 0.028 & 0.000 & - & & & & \\
\hline 8 & & MK84328o & 0.251 & $0.25^{2}$ & 0.238 & 0.034 & 0.036 & 0.012 & 0.010 & - & & & \\
\hline 9 & & MK843281 & 0.254 & 0.255 & 0.236 & 0.026 & 0.028 & 0.010 & 0.007 & 0.008 & - & & \\
\hline 10 & & MK843282 & $0.25^{6}$ & $0.25^{6}$ & 0.233 & 0.025 & 0.027 & 0.010 & 0.007 & 0.012 & 0.003 & - & \\
\hline 11 & & MK843283 & 0.251 & $0.25^{2}$ & 0.233 & 0.025 & 0.027 & 0.010 & 0.007 & 0.012 & 0.003 & 0.003 & - \\
\hline 12 & & MK843284 & 0.253 & 0.254 & 0.236 & 0.024 & 0.026 & 0.008 & 0.005 & 0.010 & 0.002 & 0.002 & 0.002 \\
\hline 13 & & MK843285 & 0.253 & 0.254 & 0.236 & 0.024 & 0.026 & 0.008 & 0.005 & 0.010 & 0.002 & 0.002 & 0.002 \\
\hline 14 & & MK843286 & 0.254 & 0.255 & 0.237 & 0.024 & 0.028 & 0.010 & 0.007 & 0.012 & 0.003 & 0.003 & 0.003 \\
\hline 15 & & MK843287 & 0.255 & $0.25^{6}$ & 0.231 & 0.032 & 0.033 & 0.016 & 0.013 & 0.018 & 0.009 & 0.009 & 0.009 \\
\hline 16 & & MK843288 & 0.256 & 0.256 & 0.233 & 0.020 & 0.026 & 0.012 & 0.008 & 0.012 & 0.005 & 0.005 & 0.005 \\
\hline 17 & & MK843289 & 0.250 & 0.251 & 0.232 & 0.022 & 0.026 & 0.015 & 0.012 & 0.017 & 0.008 & 0.008 & 0.008 \\
\hline 18 & & MK84329o & 0.247 & 0.250 & 0.228 & 0.017 & 0.022 & 0.012 & 0.008 & 0.017 & 0.008 & 0.008 & 0.008 \\
\hline 19 & & MK843291 & 0.246 & 0.247 & 0.231 & 0.017 & 0.020 & 0.017 & 0.013 & 0.019 & 0.013 & 0.013 & 0.013 \\
\hline 20 & & MK843292 & 0.244 & 0.245 & 0.229 & 0.020 & 0.021 & 0.015 & 0.012 & 0.013 & 0.008 & 0.008 & 0.008 \\
\hline 21 & & MK843293 & 0.244 & 0.246 & 0.223 & 0.012 & 0.014 & 0.014 & 0.010 & 0.019 & 0.010 & 0.010 & 0.010 \\
\hline 22 & & MK843294 & 0.244 & 0.245 & 0.229 & 0.015 & 0.019 & 0.020 & 0.017 & 0.019 & 0.013 & 0.013 & 0.013 \\
\hline 23 & & MK843295 & 0.230 & 0.232 & 0.224 & 0.008 & 0.008 & 0.027 & 0.024 & 0.029 & 0.024 & 0.024 & 0.024 \\
\hline 24 & $\begin{array}{l}\text { Per. } \\
\text { incertus }\end{array}$ & MK843296 & 0.369 & 0.371 & 0.353 & 0.343 & 0.337 & 0.355 & 0.353 & $0.35^{8}$ & 0.353 & $0.35^{2}$ & $0.35^{2}$ \\
\hline 25 & Per. & MK843296 & 0.415 & 0.407 & 0.390 & 0.347 & 0.349 & 0.363 & $0.35^{8}$ & 0.362 & $0.35^{8}$ & $0.35^{2}$ & 0.354 \\
\hline 26 & subcorallum & MK843296 & 0.415 & 0.407 & 0.390 & 0.347 & 0.349 & 0.363 & $0.35^{8}$ & 0.362 & $0.35^{8}$ & $0.35^{2}$ & 0.354 \\
\hline 27 & & MK843297 & 0.397 & 0.393 & 0.379 & 0.342 & 0.344 & 0.360 & 0.356 & 0.360 & 0.355 & 0.349 & $0.35^{2}$ \\
\hline 28 & Pal. pottsi & JX185714 & 0.230 & 0.310 & 0.290 & 0.296 & 0.298 & 0.323 & 0.321 & 0.323 & 0.321 & 0.318 & 0.318 \\
\hline
\end{tabular}




\begin{tabular}{|c|c|c|c|c|c|c|c|c|c|c|c|c|c|c|c|}
\hline 12 & 13 & 14 & 15 & 16 & 17 & 18 & 19 & 20 & 21 & 22 & 23 & 24 & 25 & 26 & 27 \\
\hline
\end{tabular}

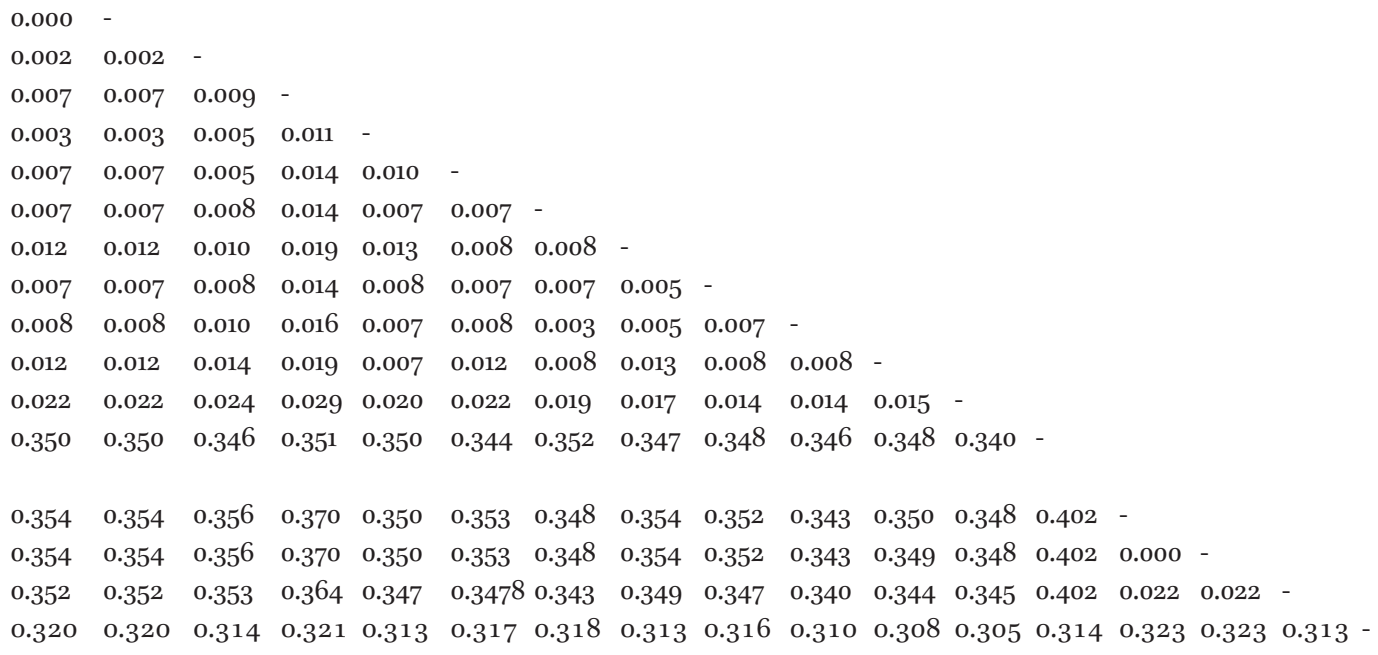


TABLE 3 Previous (p) and new (n) records of mushroom corals (Fungiidae) acting as host for Periclimenes shrimps according to Hoeksema et al. (2012) and the present results.

\begin{tabular}{llllll}
\hline & P. diversipes & P. goniopora & P.jugalis & P. watamuae & $\begin{array}{l}\text { P. subcorallum } \\
\text { sp. nov. }\end{array}$ \\
\hline Ctenactis crassa & $\mathrm{p}$ & - & - & $\mathrm{n}$ & - \\
Ctenactis echinata & - & $\mathrm{p}$ & - & $\mathrm{n}$ & - \\
Danafungia horrida & - & - & - & $\mathrm{n}$ & - \\
Danafungia scruposa & $\mathrm{n}$ & - & - & $\mathrm{n}$ & - \\
Fungia fungites & $\mathrm{n}$ & $\mathrm{p}$ & - & - & - \\
Halomitra pileus & $\mathrm{n}$ & - & - & $\mathrm{p}, \mathrm{n}$ & $\mathrm{n}$ \\
Heliofungia actiniformis & $\mathrm{p}, \mathrm{n}$ & - & $\mathrm{p}$ & $\mathrm{p}, \mathrm{n}$ & $\mathrm{n}$ \\
Herpolitha limax & $\mathrm{n}$ & - & - & $\mathrm{p}, \mathrm{n}$ & - \\
Lithophyllon concinna & $\mathrm{n}$ & - & - & - & - \\
Lithophyllon repanda & $\mathrm{n}$ & $\mathrm{p}$ & - & - & - \\
Lobactis scutaria & $\mathrm{n}$ & - & - & - & - \\
Pleuractis gravis & - & - & - & $\mathrm{n}$ & - \\
Polyphyllia talpina & - & - & - & $\mathrm{p}$ & - \\
Sandalolitha robusta & $\mathrm{n}$ & $\mathrm{p}$ & - & - & - \\
\hline
\end{tabular}

major second pereiopod of $P$. diversipes (fig. 2) is $2-3$ times as long as its distal width whereas it is as long as its distal width in $P$. watamuae (fig. 3); 2) the carpus of the minor second pereiopod of $P$. diversipes (fig. 4) is $3-4$ times as long as its distal width whereas it is $1.5^{-2}$ times as long as its distal width in $P$. watamuae (fig. 5).

\subsection{Colour}

On the basis of molecular analyses, colour differences between $P$. diversipes and $P$. watamuae were determined. Periclimenes diversipes has oblong oblique red spots on carapace and abdomen and faint transverse white bands on the abdominal segments (fig. 6) whereas P. watamuae has longitudinal red lines over carapace and abdomen, a more distinct transverse white band on the posterior part of the carapace, dorsolateral white spots on both sides on the second abdominal segment, and a clear transverse white band at the level of the fourth abdominal segment (fig. 7A, B).

\subsection{Host range}

With the use of the distinguishing features on the second chelipeds, a larger collection of symbiotic shrimps from mushroom corals could be identified (table 1). Based on earlier records and the present results, $P$. diversipes was found associated with nine mushroom coral species, all of which except one represent new host records (table 3): Danafungia scruposa (Klunzinger, 1879), Fungia fungites, Halomitra pileus, Heliofungia actiniformis, Herpolitha limax, Lithophyllon concinna (Verrill, 1864), L. repanda, Lobactis scutaria 


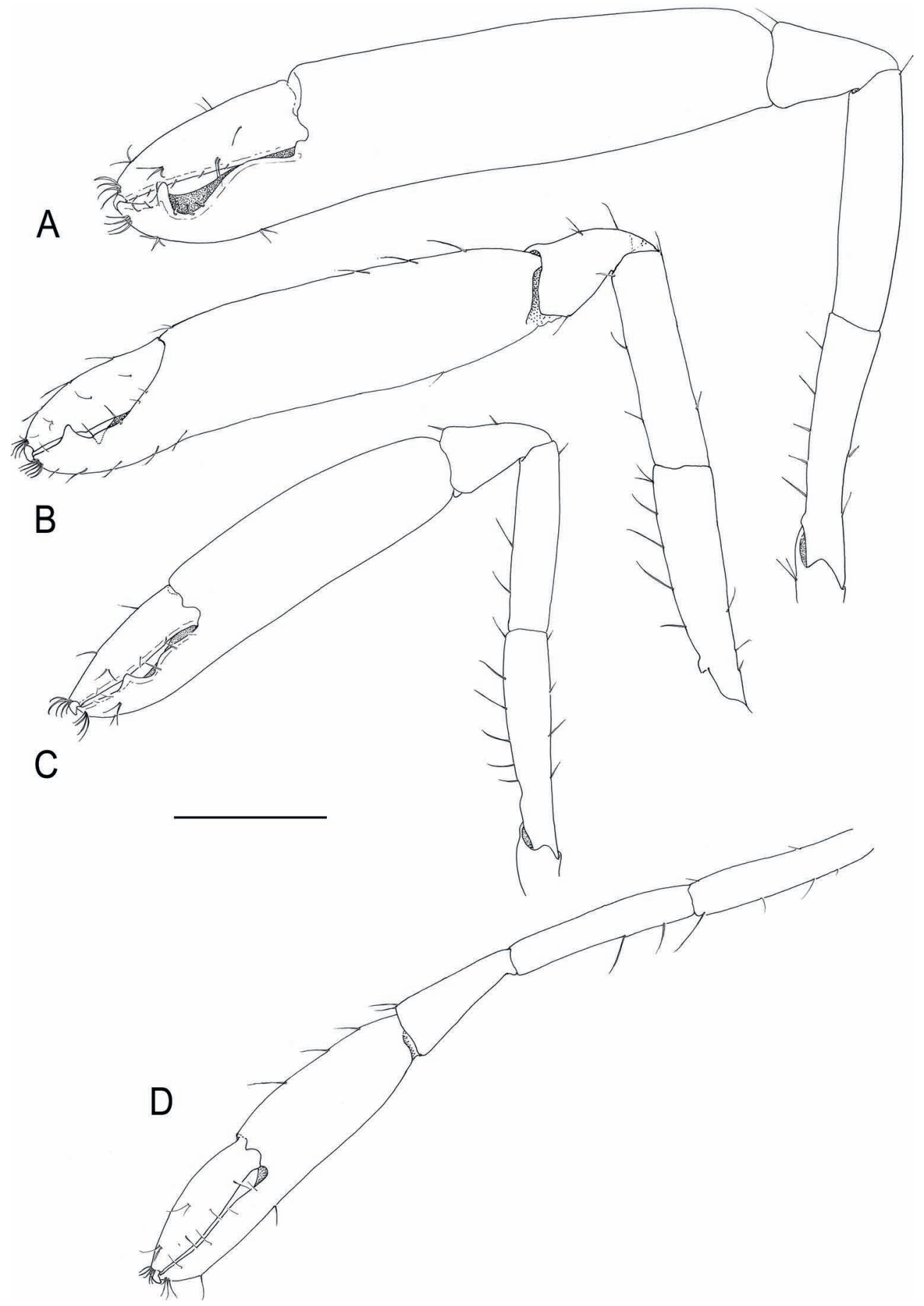

FIGURE 2 Periclimenes diversipes Kemp, 1922, major second pereiopod. A, ovigerous female pocl. 1.63 mm, left P2, RMNH.CRUS.D.57551, GenBank MK843282; B, ovigerous female pocl. 1.25 mm, right P2, RMNH. CRUS.D.57547, GenBank MK843284; C, female pocl. 1.25 mm, left P2, RMNH.CRUS.D.57548, GenBank MK843295; D, male, pocl 1.38 mm, right P2, RMNH.CRUS.57553, GenBank MK843283.

Scale bar $=0.4 \mathrm{~mm}$. 
A
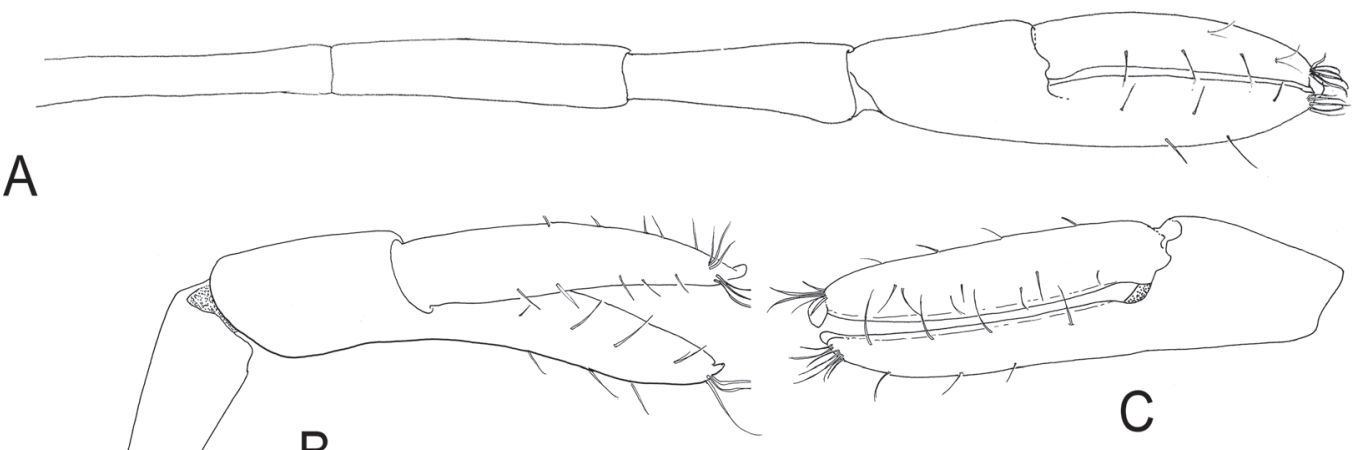

B

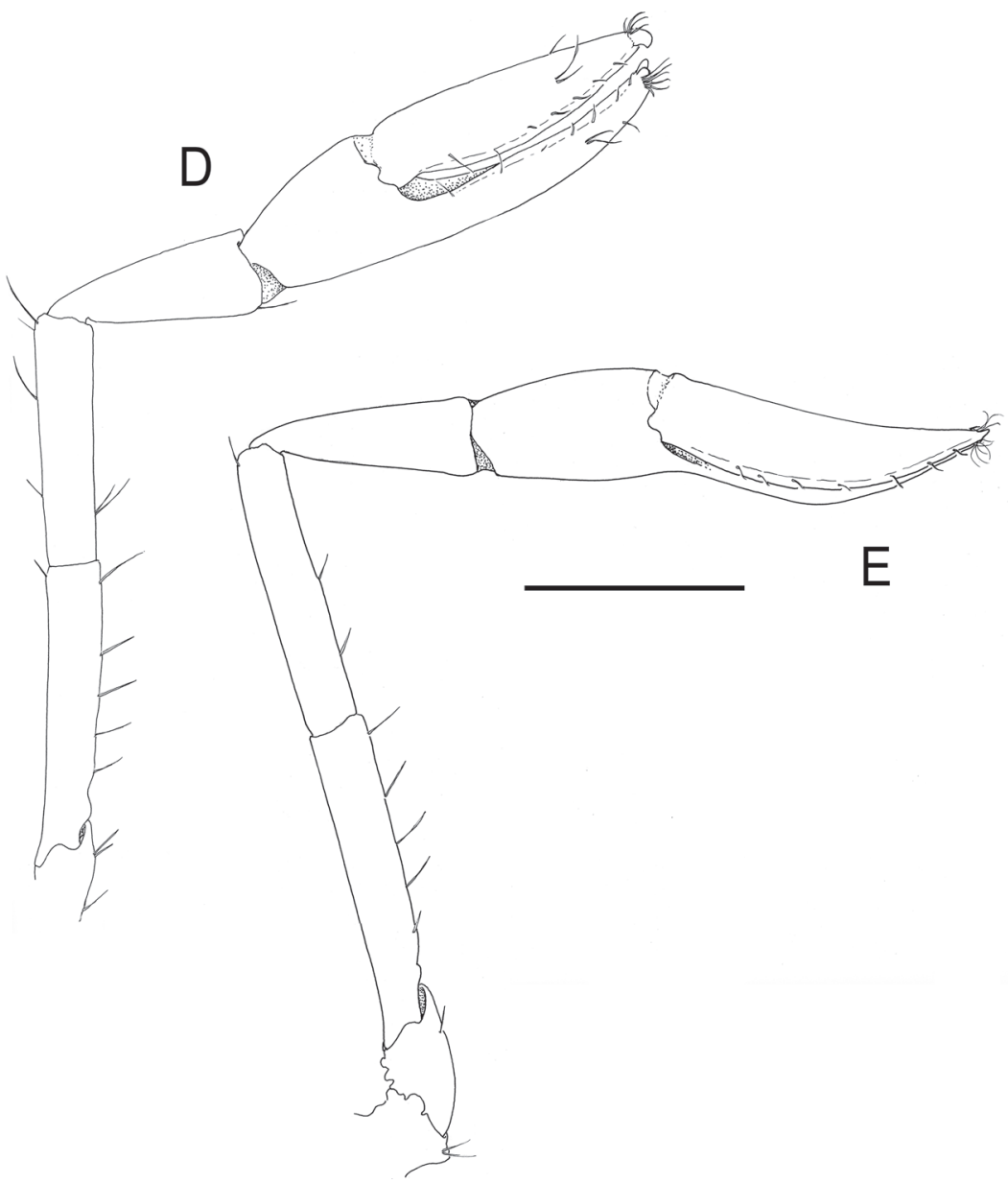

FIGURE 3 Periclimenes diversipes Kemp, 1922, minor second pereiopod. A, male, pocl 1.38 mm, left P2,

RMNH.CRUS.D.57553, GenBank MK843283; B, ovigerous female pocl. $1.25 \mathrm{~mm}$, left P2, RMNH.

CRUS.D.57547, GenBank MK843284; C, idem, lateral aspect chela; D, female pocl. $1.25 \mathrm{~mm}$, right P2,

RMNH.CRUS.D.57548, GenBank MK843295; E, ovigerous female pocl. $1.63 \mathrm{~mm}$, right P2, RMNH.

CRUS.D.57551, GenBank MK843282. Scale bar $=0.4 \mathrm{~mm}$. 


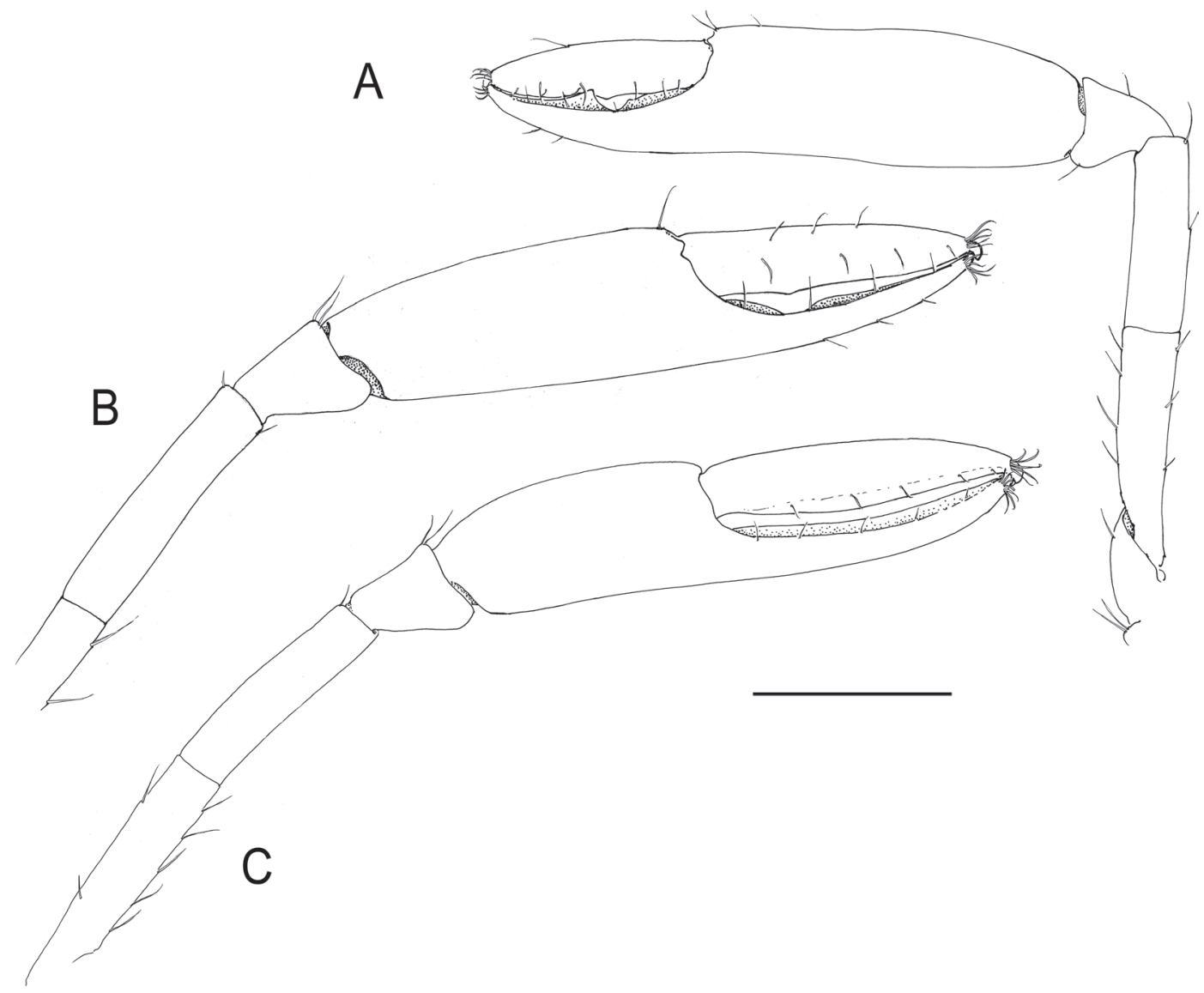

FIgURE 4 Periclimenes watamuae Bruce, 1976a, major second pereiopod. A, male, pocl 1.oo mm, right $\mathrm{P}_{2}, \mathrm{RMNH}$. CRUS.D.57574, LEM.36; B, ovigerous female, pocl 1.45 mm, left P2, RMNH.CRUS.D.57574, LEM.36; C, ovigerous female, pocl. 1.40 mm, left P2, RMNH.CRUS.D.57574, GenBank MK843275. Scale bar $=0.4 \mathrm{~mm}$.

(Lamarck, 1801), and Sandalolitha robusta. Periclimenes watamuae was found associated with seven mushroom coral species of which five constitute new host records (table 3): Ctenactis crassa, C. echinata, Danafungia horrida (Dana, 1846), D. scruposa, Heliofungia actiniformis, Herpolitha limax, and Pleuractis gravis (Nemenzo, 1955). The new Periclimenes species was found on Halomitra pileus and Heliofungia actiniformis (table 3).

The closely related $P$. diversipes and $P$. watamuae are both associated with a wide range of mushroom corals, consisting of ten and nine species, respectively (table 3 ).
They have three mushroom coral species in common that act as hosts. However, no genetic structuring within this shrimp species with regards to host choice could be detected.

The mushroom coral cladogram (fig. 8) shows that none of the Periclimenes species has ever been recorded from an attached mushroom coral species $(\mathrm{A}=$ attached) and from species with a maximum recorded size $<15 \mathrm{~cm}(\mathrm{~S}=$ small $)$. Coral species of the genus Cycloseris fit in these categories and are therefore not recorded as hosts. The host species can be found among free-living, coral species with a maximum 


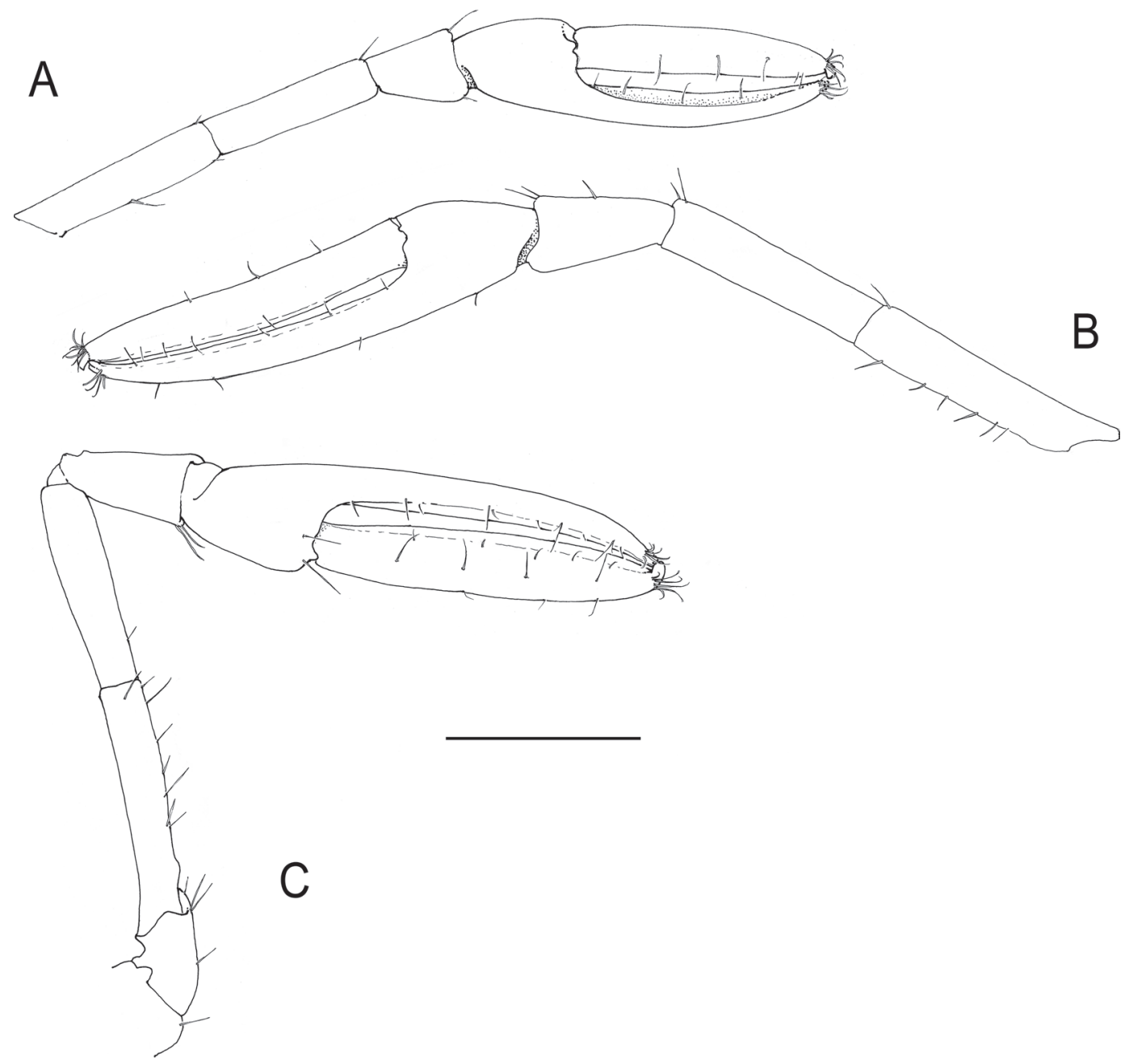

FIgURe 5 Periclimenes watamuae Bruce, 1976a, minor second pereiopod. A. male, 1.00 mm, left $\mathrm{P}_{2}$, RMNH.CRUS.D.57574, LEM.36; B, ovigerous female, pocl 1.45 mm, right P2, RMNH.CRUS.D.57574, LEM.36; C, ovigerous female, pocl. 1.40 mm, right P2, RMNH.CRUS.D.57574, GenBank MK843275. Scale bar $=0.4 \mathrm{~mm}$.

diameter $>15 \mathrm{~cm}$, which together do not form a monophyletic group within the Fungiidae (fig. 8).

\subsection{Systematic account}

Family Palaemonidae Rafinesque, 1815

Genus Periclimenes O.G. Costa, 1844

Periclimenes subcorallum Fransen \& Rauch, sp. nov.

(Figs. 9-13) http://zoobank.org/urn:lsid:zoobank.org: act: 5129C847-F357-4363-B5A8-96869C3 FD144 Material examined. Indonesia, NE Sulawesi, Lembeh Strait. Ovigerous female holotype (pocl. 1.50mm) MZB Cru 4968; 4 ovigerous female paratypes (pocl. $1.45^{-1.78}$ $\mathrm{mm}, 1$ non-ovigerous female (pocl. $1.38 \mathrm{~mm}$ ), 2 males (pocl. 1.35 and $1.40 \mathrm{~mm}$ ) RMNH. CRUS.D.57575: stn LEM.24, Tanjung Pandea, $1^{\circ} 23^{\prime} 5^{2}$ "N $125^{\circ}{ }^{\circ} 9^{\prime} 58^{\prime \prime E}$, 11.ii.2012, depth 


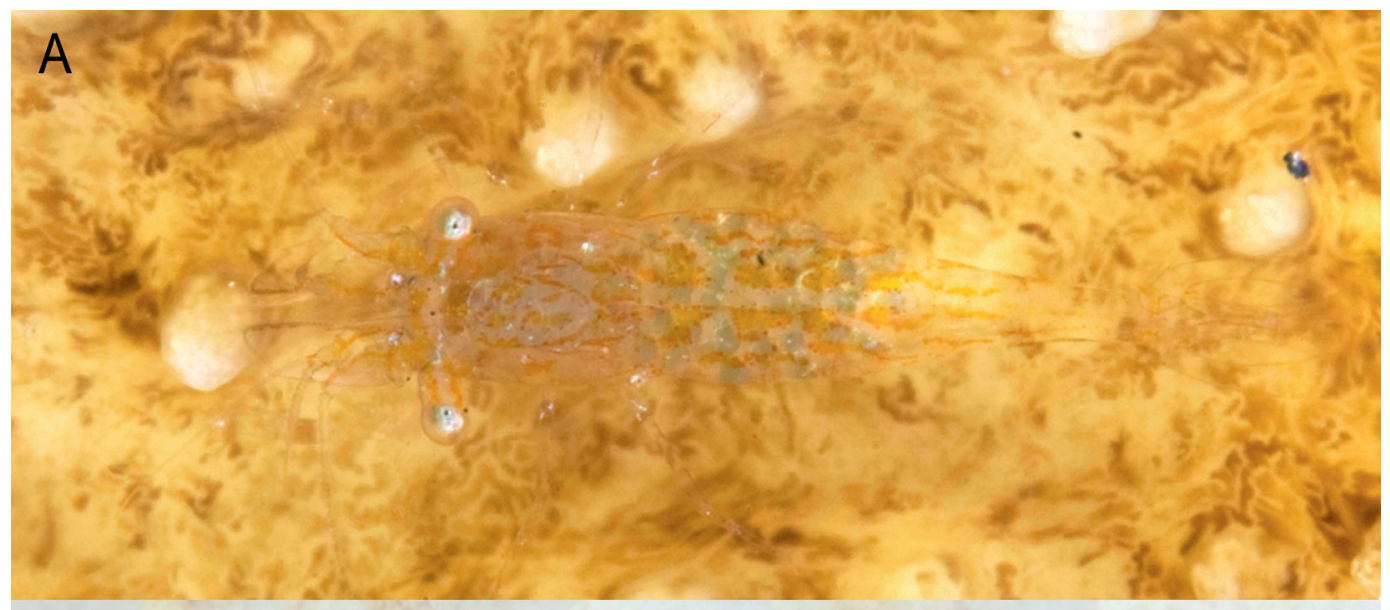

B

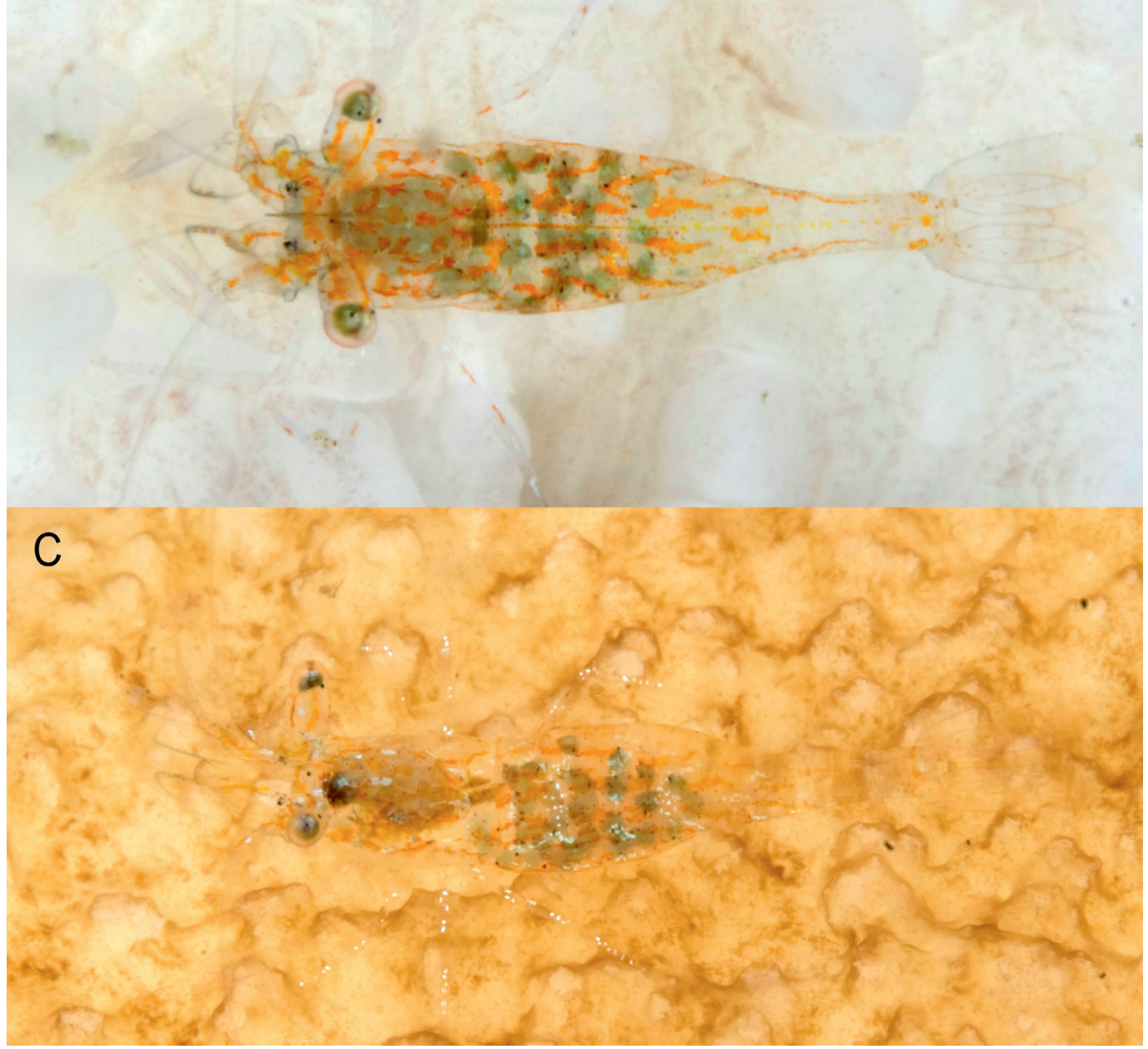

FIGURE 6 Periclimenes diversipes Kemp, 1922, ovigerous female. A, stn LEM.o7, on Danafungia scruposa; B, stn LEM.o7, on Lithophyllon repanda; C, stn LEM.18, on Sandalolitha robusta. 


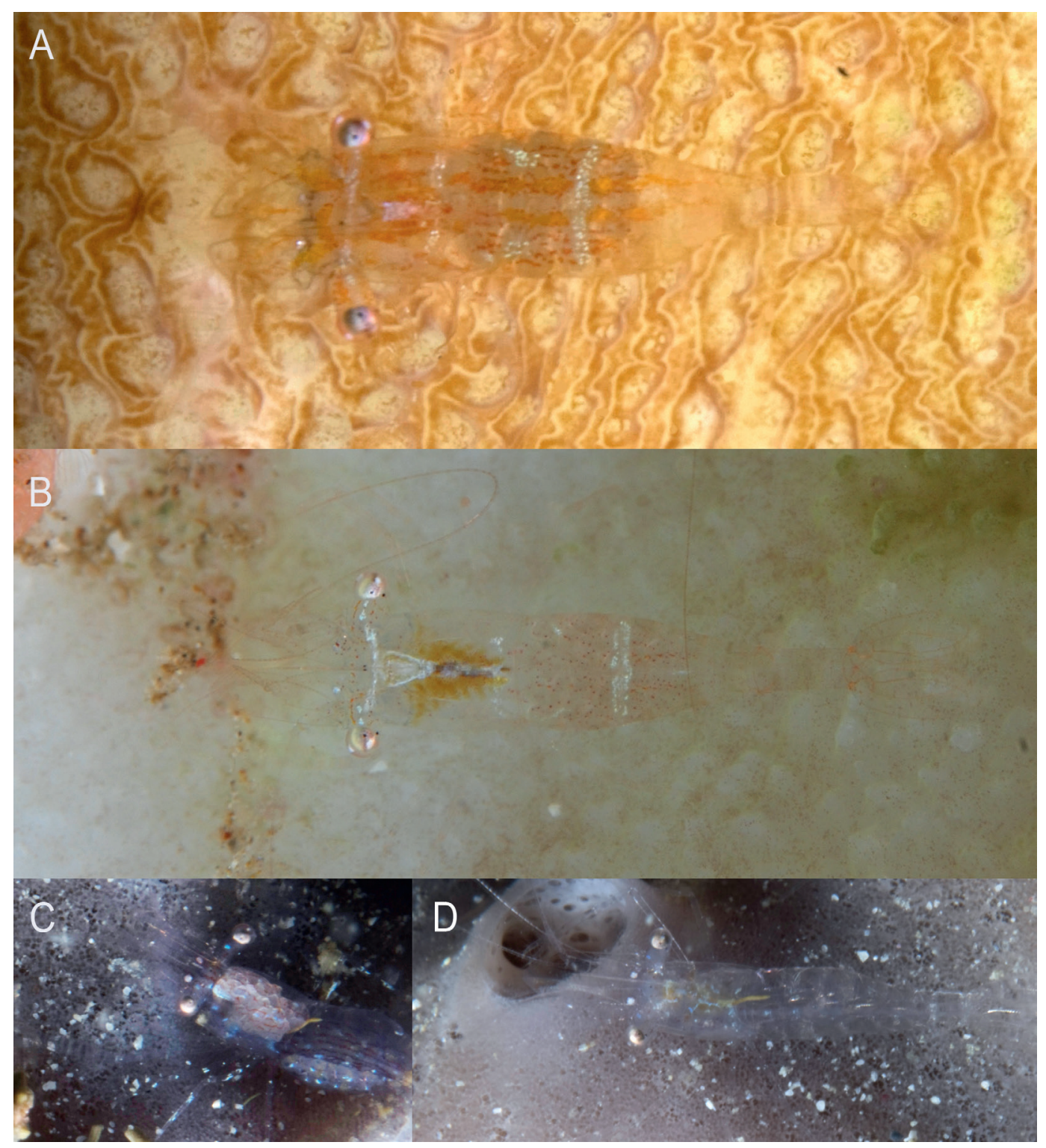

FIgURE 7 A-B, Periclimenes watamuae Bruce, 1976a, ovigerous female; C-D, Periclimenes incertus Borradaile, 1915. A, stn LEM.26, on Lobactis scutaria; B, stn TER.16, on Polyphyllia talpina; C-D, stn SEM.48, on sponge: $\mathrm{C}$, ovigerous female; $\mathrm{D}$, male.

$7 \mathrm{~m}$, on Heliofungia actiniformis, coll. Cessa Rauch. - 1 ovigerous female (pocl. $1.25 \mathrm{~mm}$ ) and 1 male (pocl. $1.18 \mathrm{~mm}$ ) paratypes, RMNH. CRUS.D.57577: stn LEM.28, Desa Pandean, $1^{\circ} 24^{\prime} 21$ "N125 ${ }^{\circ}{ }^{\prime}{ }^{\prime} 5^{2}$ "E, 14.ii.2012, depth $13.2 \mathrm{~m}$, on Heliofungia actiniformis, coll. Cessa Rauch. 1 ovigerous female (pocl. $1.63 \mathrm{~mm}$ ) paratype,
RMNH.CRUS.D.57576: stn LEM.28, Desa Pandean, $1^{\circ} 24^{\prime} 21$ "N $125^{\circ} 10^{\prime} 5^{2}$ "E, 14.ii.2012, depth 20 m, on Halomitra pileus, coll. C.H.J.M. Fransen.

Comparative material examined. Periclimenes incertus Borradaile, 1915 (figs. 8C, D, 14-16). Malaysia, Sabah, Semporna. 27 ovigerous females 2 non-ovigerous females, 15 males 


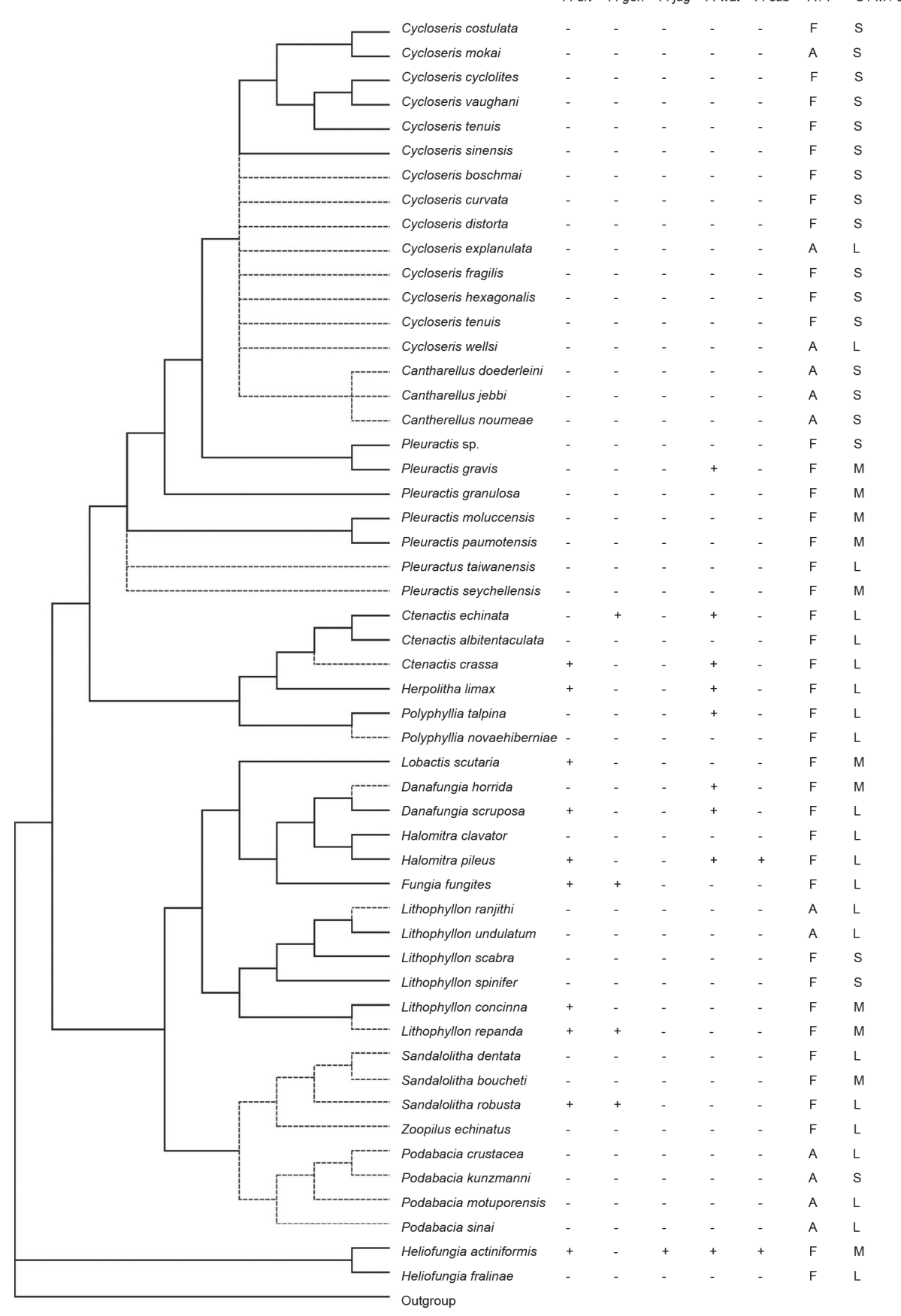

FIGURE 8 Mushroom coral cladogram based on published phylogenetic analyses (Gittenberger et al., 2011; Benzoni et al., 2012). The host relations of five Periclimenes species (table 3) are projected on the phylogeny reconstruction as well as the morphological traits "attached vs. free-living mode of life" (A = attached / $\mathrm{F}=$ free-living) and maximum recorded corallum size (Hoeksema, 1991, 2012a, 2014; Gittenberger et al., 2011) with size categories ( $\mathrm{S}=$ small, $<15 \mathrm{~cm} ; \mathrm{M}=$ medium, $\geq 15 \mathrm{~cm}$ and $<25 \mathrm{~cm} ; \mathrm{L}=$ large, $\geq 25 \mathrm{~cm}$ ). $P$. div $=$ Periclimenes diversipes, $P$. gon = Periclimenes gonioporae, $P$.jug $=$ Periclimenes jugalis, $P$. wat $=$ Periclimenes watamuae, $P$. sub = Periclimenes subcorallum sp. nov. 


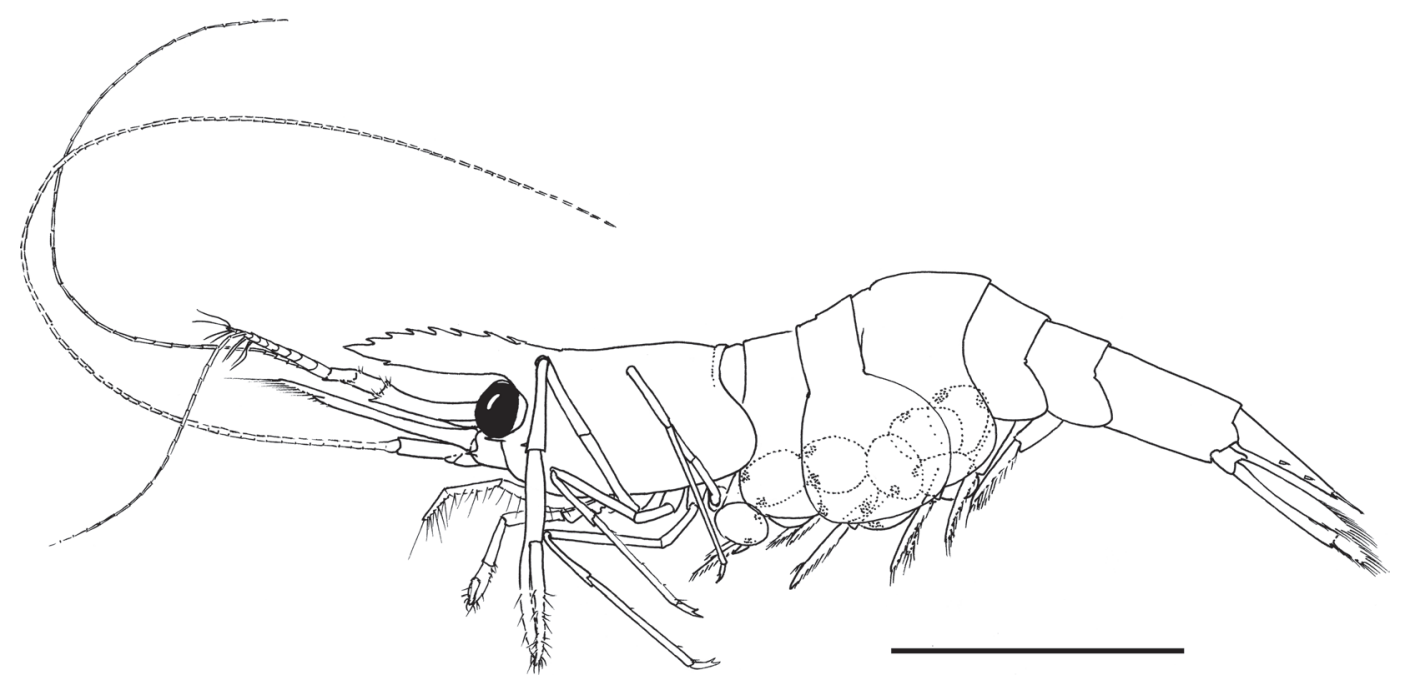

FIGURE 9 Periclimenes subcorallum sp. nov., ovigerous female holotype (pocl. 1.50 mm), MZB CRU 4968. Habitus. Scale $=2 \mathrm{~mm}$.

and 6 juveniles (pocl. 1.00-2.08 mm), RMNH. CRUS.D.53946: stn SEM.48, Timbun Mata Isl.,

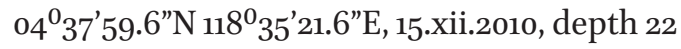
$\mathrm{m}$, on grey encrusting sponge, coll. C.H.J.M. Fransen.

Description. Small-sized, rather slender palaemonid shrimp, with slender pereiopods (fig. 9).

Carapace smooth. Rostrum (fig. 10A, B) well developed, reaching halfway distal segment of antennular peduncle; lamina deep, lateral carina indistinct, situated near to proximally slightly convex ventral margin with 1 subdistal tooth; convex posterior part of ventral margin with double row of plumose setae; dorsal margin convex, elevated, strongly compressed, with 6 subequal teeth, posteriormost situated at level of posterior margin of orbit; 2-3 plumose setae just in front of each dorsal teeth. Supra-orbital spines absent. Inferior orbital angle well developed, produced, angular in lateral view. Antennal spine of moderate size, marginal, situated below inferior orbital angle. Hepatic spine about as large as antennal spine, situated well behind level of posterior orbital margin and slightly below level of antennal spine. Antero-lateral angle of carapace blunt, not produced.

Abdominal segments (fig. 9) smooth. Third segment not produced posterodorsally. Pleura all broadly rounded. Sixth abdominal segment 2.3 times as long as fifth (relatively longer in smaller specimens), posteroventral angle feebly produced, rounded, posterolateral angle acute.

Telson (fig. 14A) 0.1 times as long as sixth abdominal segment and 3.0 times longer than anterior width; lateral margins converge posteriorly; two pairs of submarginal dorsal spines present at 0.59 and 0.82 of telson length, posterior margin (fig. 14B) rounded, 0.36 of anterior width, with three pairs of spines. Lateral spines short, as long as dorsal spines. Intermediate spines well developed, about 0.22 of telson length, 2.2 times length of submedian spines.

Eyes (fig. 10A, B) well developed. Cornea globular, with distinct accessory pigment spot posterodorsally. Eyestalks almost twice as long as proximal width, slightly swollen proximally.

Antennular peduncle (fig. 10A, B) exceeding tip of rostrum by half of distal segment. 
Proximal segment long, slender, 2.5 times longer than wide; stylocerite slender, acute, reaching almost to middle of segment; lateral margin straight, anterolateral margin produced, rounded, with distolateral tooth and row of setae; ventral margin with small submedian tooth at about 0.4 of length of segment. Statocyst containing granular statolith. Intermediate and distal segments short, together equal to 0.48 of proximal segment length. Upper flagellum biramous, with the first $5^{-7}$ segments fused; short ramus with 3 segments; aesthetascs present on short free ramus only. Longer free ramus long and slender, with about 25 segments. Lower flagellum very long and slender, almost twice as long as longer free ramus of upper flagellum.

Antennal basicerite (fig. 10B) with strong lateral tooth. Ischiocerite and merocerite normal. Carpocerite slender, reaching 0.4 of length of scaphocerite. Scaphocerite long, rather slender, with lamella distinctly overreaching distal margin of antennular peduncle. Lateral border straight, ending in acute large distolateral tooth. Lamella extending beyond distolateral tooth, feebly angulated distomedially, about 3.5 times longer than broad, with greatest width at about one half of its length.

Epistome, labrum and paragnath without special features.

Second thoracic sternite (fig. 10G) formed into broad triangular medially rounded process between second maxillipeds.

Third thoracic sternite unarmed (fig. 10G).

Fourth thoracic sternite with broad medial triangular plate with median notch posteromedial of first pereiopods (fig. $10 \mathrm{G}$ ).

Fifth thoracic sternite with shallow lateral plates posteromedial of second pereiopods.

Sixth to eighth thoracic sternites broad, unarmed.

Mandible (fig. 10C) with cylindrical molar process with blunt teeth on strong chewing surface, with 2 short bands of few setae subdistally. Incisor process slender, with 3 well developed teeth distally, of which later almost slightly enlarged. Mandible without palp. Maxillula (fig. $10 \mathrm{D}$ ) with upper lacinia rectangular with rows of few serrulate spines and slender setae medially; lower lacinia slender, with few serrulate setae distally; palp bilobed, medial lobe with single short recurved simple seta.

Maxilla (fig. 10E) with short tapering palp with few plumose setae laterally. Basal endite bilobed, distal lobe slightly broader than proximal lobe, both lobes with row of about 10 minutely serrate setae medially. Coxal endite obsolete, median margin convex, without setae. Scaphognathite normal, widest centrally, about 2.9 times longer than broad, with marginal plumose setae.

First maxilliped (fig. 10F) with short, slender, tapering palp without setae. Basal region broad, not distinctly separated from the coxal region, with median margin provided with setulose and slender simple setae. Coxal region strongly convex with few minutely serrulate setae and 2 large long plumose setae medially. Caridean lobe with coarsely setulose plumose marginal setae. Flagellum of exopod well developed with 4 long plumose distal setae. Epipod bilobed.

Second maxilliped (fig. 11A) with dactylar segment narrow, about 3.0 times longer than wide, straight medially, densely fringed with numerous coarsely serrulate, spiniform, and long curled finely serrulate setae medially. Propodal segment longer than dactylar segment twice as long as wide, with distomedial margin not produced, with few long serrulate setae. Carpus short, unarmed. Merual segment short, not excavate, without setae. Ischium completely fused to basis. Basis with long slender exopod about as long as length of endopod, with 4 long plumose setae distally and few shorter plumose seta subdistally. Coxa slightly produced medially, with 2 long setae medially, small oblong epipod laterally. 


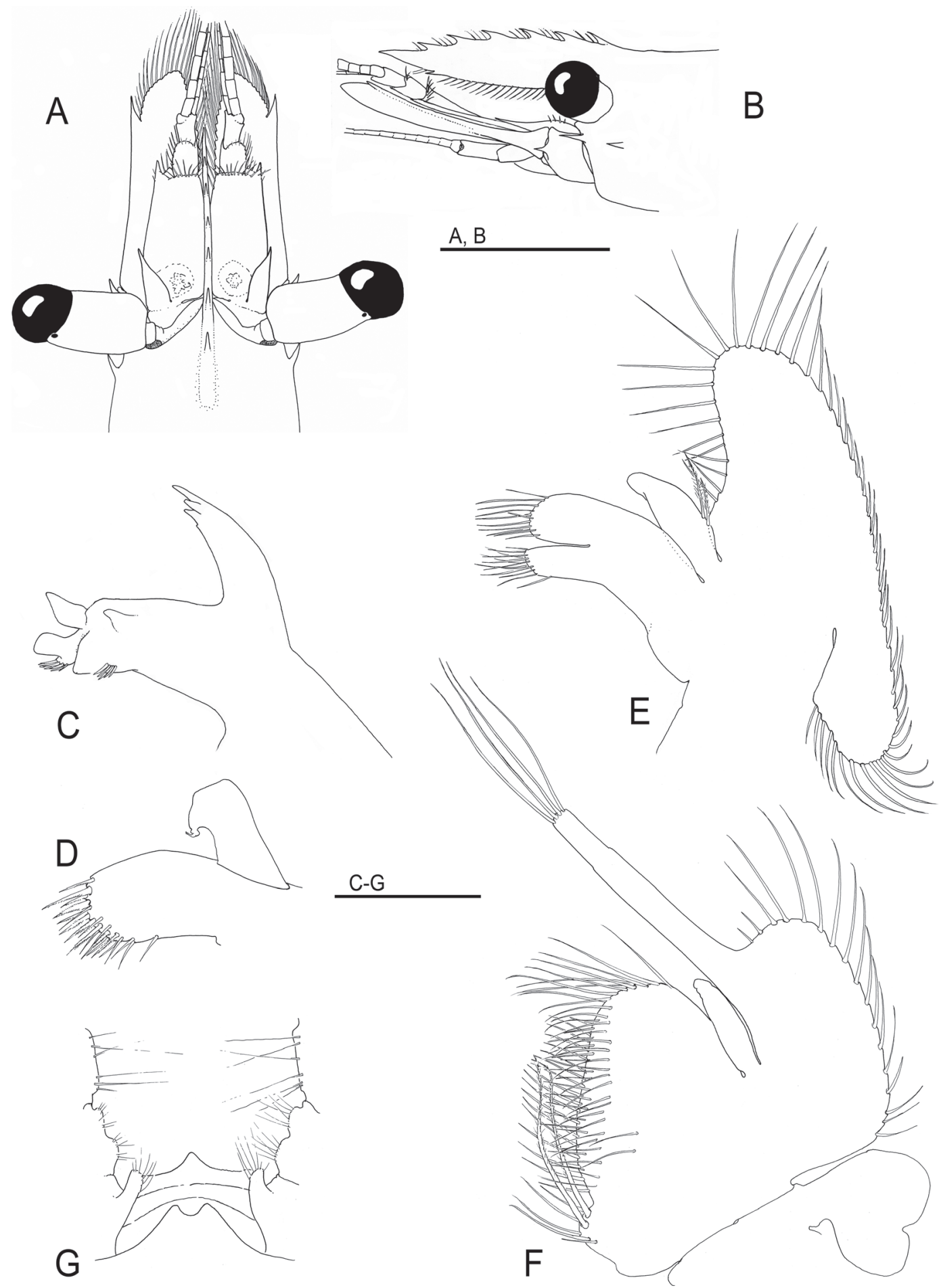

FIGURE 10 Periclimenes subcorallum sp. nov., ovigerous female paratype (pocl. $1.45 \mathrm{~mm}$ ), RMNH.CRUS.D.57575. A, rostrum and anterior appendages, dorsal view; B, idem, lateral view; C, left mandible; D, left maxillula; E, left maxilla; F, left first maxilliped; G, fourth thoracic sternites and proximal segments of first

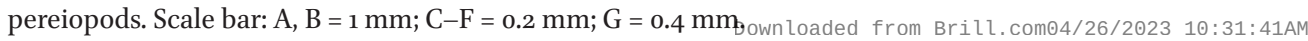


Third maxilliped (fig. $11 \mathrm{~B}$ ) slender. Terminal segment 3.5 times longer than proximal width, 0.57 of length of penultimate segment, with rows of short serrulate setae medially and longer simple setae ventrolaterally. Penultimate segment slender, 4.12 times longer than wide with rows of long finely serrulate setae medially and ventrolaterally. Ischiomerus and basis fused, combined segment 1.50 times as long as penultimate segment, 4.78 times as long as distal wide; proximomedial margin rounded with few simple setae followed by row of 5 short serrulate setae, meral part with row of long minutely serrulate setae, with one subdistal lateral spine. Exopod reaching 0.8 of combined segment, with 4 distal and 3 subdistal plumose setae. Coxa not produced medially, with rounded lateral plate, without arthrobranch.

First pereiopod (fig. $11 \mathrm{C}$ ) slender, reaching to end of scaphocerite. Chela (fig. $11 \mathrm{E}$ ) with palm subcylindrical, straight, 1.6 times longer than wide. Fingers as long as palm, straight not subspatulate, with brushes of few setae in distal part, cutting edges entire, tips of fingers hooked. Cleaning setae present proximally on palm and distoventral part of carpus. Carpus 1.3 times length of chela, 5.0 times longer than wide. Merus slightly longer than carpus, twice length of ischium. Ischium with medial setal ridge with few long simple setae. Basis with proximomedial setal ridge and distomedial small setose process. Coxa with setose medial process (fig. 11D).

Second pereiopods (fig. 12A, D), equal in length, dissimilar. Major left second pereiopod (fig. 12A) extending beyond antennular peduncle with chela. Chela with palm subcylindrical, straight, 3.6 times as long as wide. Fingers (fig. 12B) 0.41 of palm length. Dactylus as wide as fixed finger, fingers gaping in middle, both with brushes of setae in distal part, tips (fig. $12 \mathrm{C}$ ) strongly hooked. Dactylus with cutting edge with one triangular tooth proximally of gape and one triangular tooth distally of gape, cutting edge of gape rounded, that between distal tooth and hooked tip acute. Fixed finger with three blunt teeth proximally of gape and one triangular tooth distally of gape, cutting edge of gape rounded, that between distal tooth and hooked tip acute. Carpus gradually increasing in width distally, merus and ischium unarmed, their length ratios of $0.37,0.69$ and 0.80 times length of palm. Basis with few setae medially. Coxa with small median setose process. Minor second chela (fig. 12D) with fingers 0.8 times length of subcylindrical palm, with setal brushes, fingers (fig. 12E) distally hooked, cutting edges with small triangular tooth in middle, acute distally of tooth, rounded proximally. Carpus gradually increasing in with distally; merus and ischium unarmed, their length ratios of $0.81,1.04$ and 1.26 times length of palm. Basis and coxa as in major chela.

The ambulatory pereiopods (fig. 13A, C, E) slender, similar in form, slightly increasing in length from third to fifth pereiopod, reaching with dactyli to distal margin of scaphocerite. Dactylus of third pereiopod (fig. ${ }_{13} \mathrm{~B}$ ) slender, not tapering, 0.24 of propodus length, 4.1 times as long as proximal width, with large accessory tooth reaching to 0.75 of slightly curved unguis length, accessory tooth and unguis with same proximal height; flexor margin of corpus concave with series of 5 small denticles. Propodus 12 times longer than wide, with two distoventral spines and 2 small ventral spines; distoventral spines with fine denticulation on flexor margin. Carpus, merus and ischium 0.50, 1.10 and 0.50 of propodus length, unarmed. Fourth (fig. ${ }_{13} \mathrm{C}, \mathrm{D}$ ) and fifth (fig. 13E, F) pereiopods similar as third, fourth with 3 ventral teeth, fifth with 1 ventral tooth on propodus.

Endopod of first pleopod in ovigerous female short (fig. 14D), 0.4 of length of exopod, with long plumose setae along its entire 


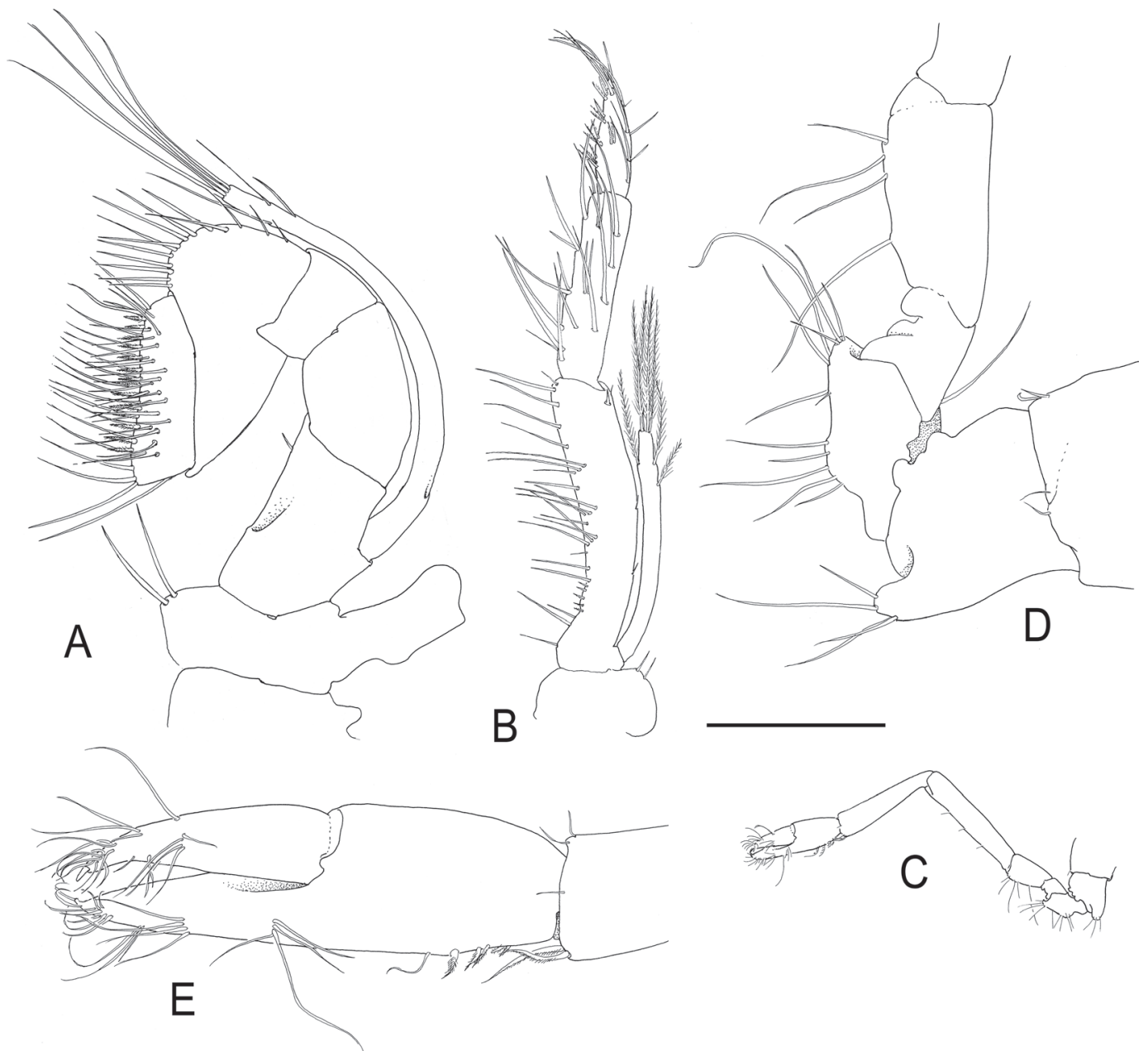

FIGURE 11 Periclimenes subcorallum sp. nov., ovigerous female paratype (pocl. 1.45 mm), RMNH.CRUS.D.57575. A, left second maxiliped; B, left third maxilliped; C, left first pereiopod; D, idem, proximal segments; E, idem, chela. Scale bar: A, D, E = $0.2 \mathrm{~mm} ; \mathrm{B}=0.4 \mathrm{~mm} ; \mathrm{C}=1 \mathrm{~mm}$.

margin. Endopod of first pleopod in male (fig. ${ }_{14} \mathrm{E}$ ) short, 0.4 times length of exopod, distally broadly rounded with median lobe, with few long plumose setae along lateral margin and about 6 very short simple setae in proximal 2/3rd of medial margin. Endopod of second pleopod in male (fig. 14F) slightly shorter length of exopod. Appendix masculina long and slender, almost twice as long as appendix interna, with five serrate long setae distally.

Uropods extending beyond tip of telson. Protopodite unarmed laterally. Exopod with lateral border almost straight, slightly setose in proximal part, terminating in a small distolateral tooth with mobile spine medially, mobile spine twice as long as distolateral tooth (fig. 14C). 


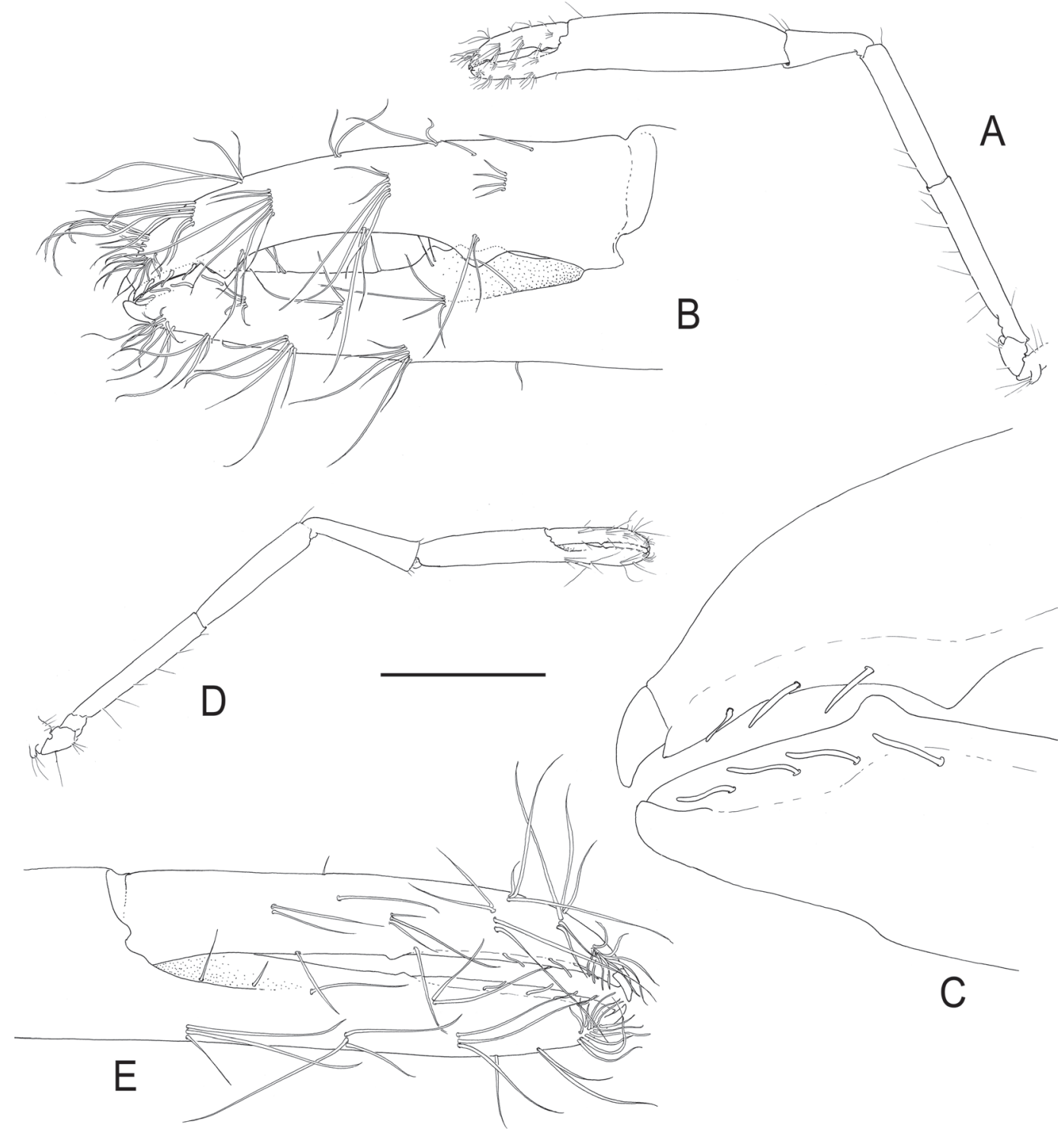

FIGURE 12 Periclimenes subcorallum sp. nov., ovigerous female paratype (pocl. 1.45 mm), RMNH.CRUS.D.57575. A, left major second pereiopod; B, idem fingers of chela; $C$, idem, tip of fingers, setae omitted; D, right minor second pereiopod; E, idem, fingers of chela. Scale bar: $\mathrm{A}, \mathrm{D}=1 \mathrm{~mm} ; \mathrm{B}, \mathrm{E}=0.2 \mathrm{~mm} ; \mathrm{C}=0.07 \mathrm{~mm}$.

About 25 eggs of ca. $0.4 \mathrm{~mm}$ in diameter present under abdomen.

Colouration. Not known.

Etymology. The epithet 'subcorallum' refers to the observed position of the shrimps in relation their hosts, underneath corals.
Host records. Heliofungia actiniformis and Halomitra pileus (Fungiidae, Scleractinia).

Systematic position. The new species belongs to the Periclimenes obscurus species group comprising: P. batei (Borradaile, 1917); P. burrup Bruce, 2007; P. delagoae Barnard, 


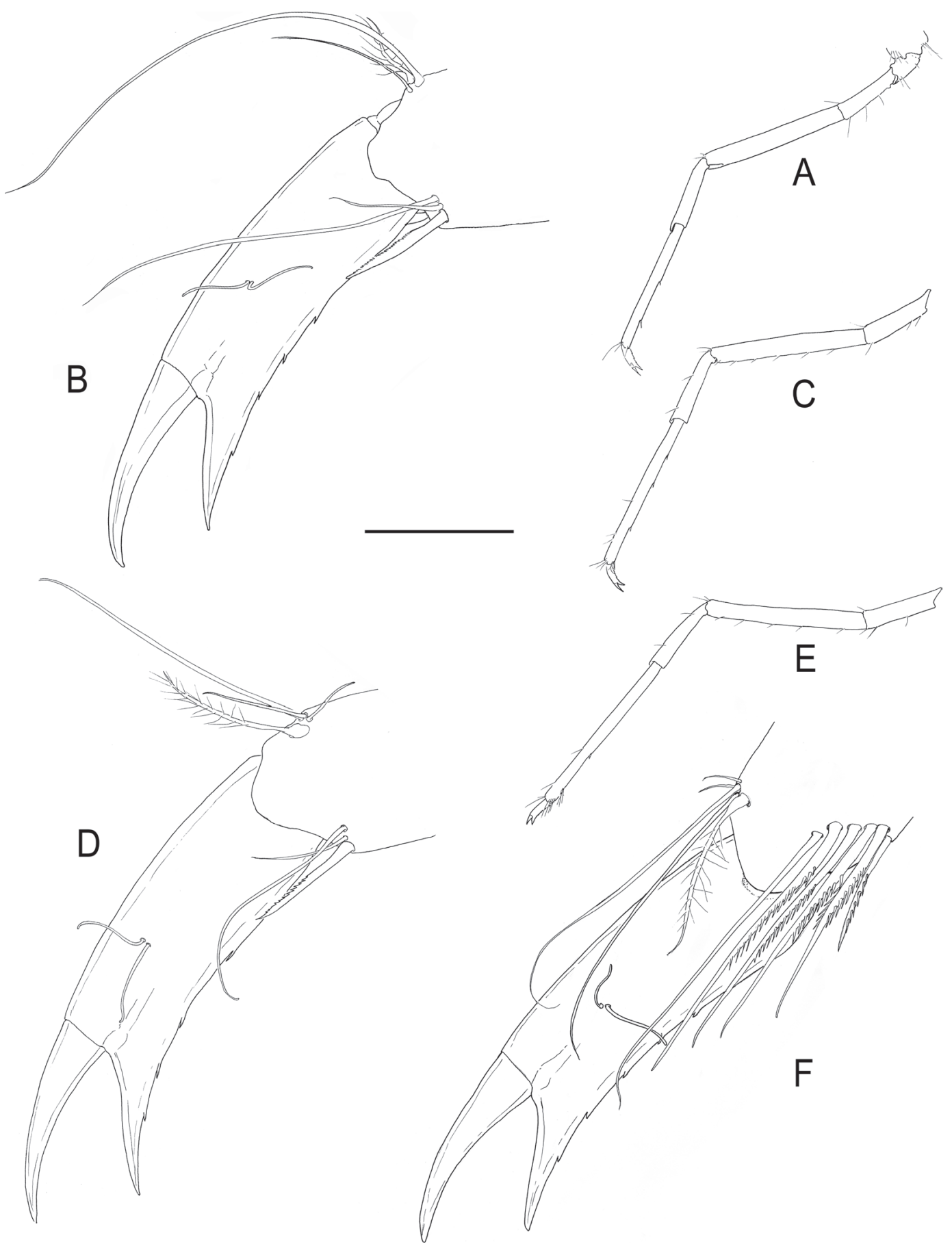

FIGURE 13 Periclimenes subcorallum sp. nov., ovigerous female paratype (pocl. 1.45 mm), RMNH.CRUS.D.57575. A, left third pereiopod; B, idem, dactylus; C, left fourth pereiopod; D, idem, dactylus; E, left fifth pereiopod; F, idem, dactylus. Scale bar: A, C, E = $1 \mathrm{~mm} ; \mathrm{B}, \mathrm{D}, \mathrm{F}=0.07 \mathrm{~mm}$. 


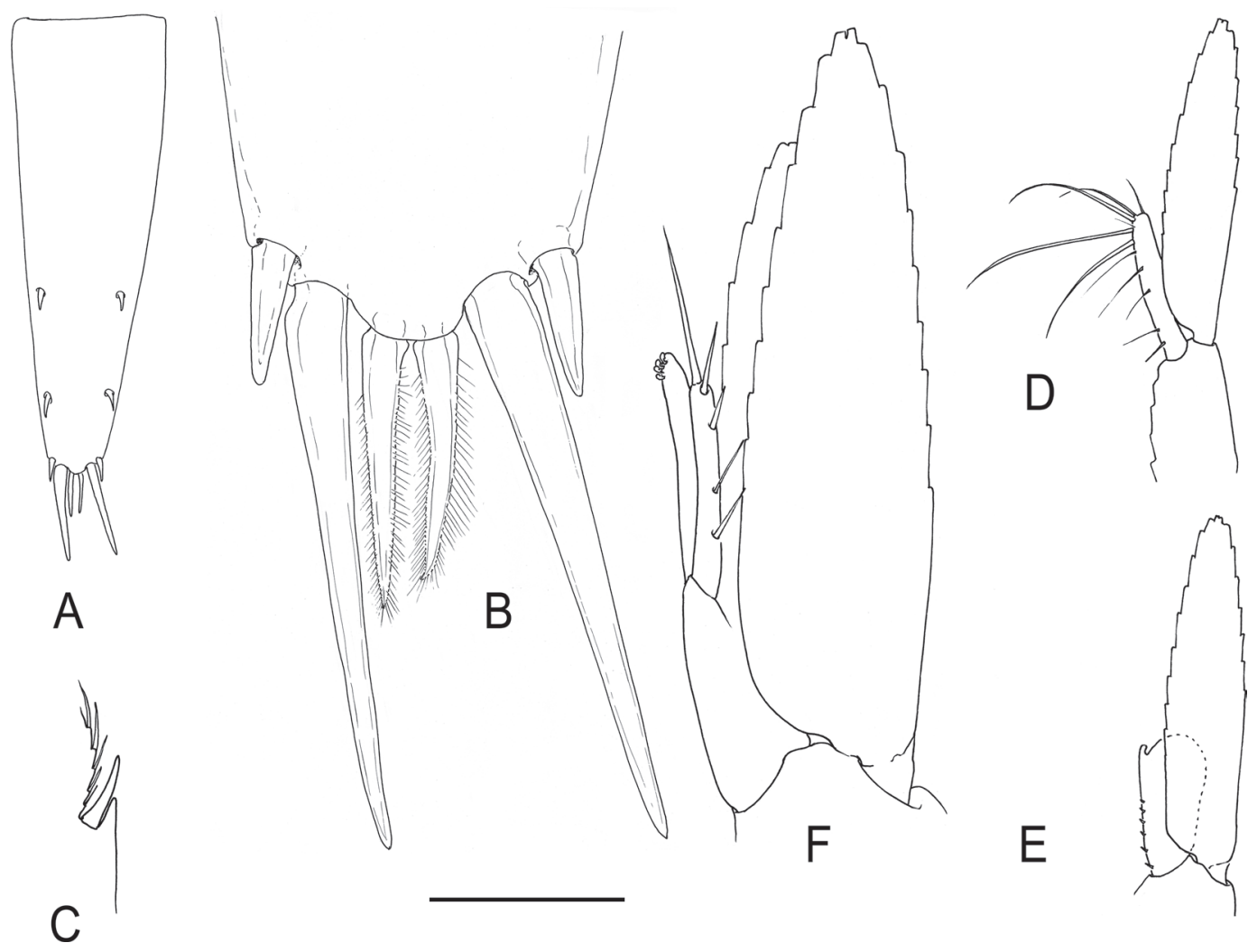

FIGURE 14 Periclimenes subcorallum sp. nov., ovigerous female paratype (pocl. 1.45 mm), RMNH.CRUS.D.57575. A, telson; B, idem, detail distal part; C, distolateral part of uropod exopod; D, right first pleiopod. Male paratype (pocl. 1.35 mm), RMNH.CRUS.D.57575. E, right first pleiopod; F, right second pleopod. Scale bar: $\mathrm{A}, \mathrm{C}, \mathrm{E}=0.4 \mathrm{~mm}$; $\mathrm{B}=0.07 \mathrm{~mm}$;, $\mathrm{F}=0.2 \mathrm{~mm}$.

1958; P. hongkongensis Bruce, 1969; P. incertus Borradaile, 1915; P. macrorhynchia Eilbracht \& Fransen, 2015; P. nomadophila Berggren, 1994; P. obscurus Kemp, 1922; P. sinensis Bruce, 1969; P. terangeri Bruce, 1998; P. toloensis Bruce, 1969; P. zevinae Ďuriš, 1990 (Bruce, 1969, 1987, 2007; Ďuriš, 1990; Berggren, 1994; Eilbracht \& Fransen, 2015). Species in the group have a well-developed, horizontal rostrum with a moderately deep lamina bearing numerous dorsal teeth and o to 2 ventral teeth; the antennal spine is well developed, a supraorbital spine is absent, the epigastric spine is present or absent; the inferior orbital angle is feebly produced; the fourth thoracic sternite is without a slender median process; the third abdominal segment is not conspicuously produced posterodorsally; the first pereiopods have simple or 'nail-like' fingers and a more or less developed setose distoventral process on the coxa; the second pereiopods are slender, unspecialized, carpus more than one third of length of palm, merus unarmed; dactylus of ambulatory pereiopods usually biunguiculate. The delimitation of the group is rather vague and seems to overlap with species from the $P$. diversipes and $P$. granulimanus species groups.

The new species is most similar to $P$. incertus Borradaile, 1915 and P. zevinae Ďuriš, 1990. Both species share the denticulate ventral margin of the corpus of the ambulatory 
pereiopods with the new species. These three species also have the accessory tooth of the dactyli of the ambulatory pereiopods as stout as or stouter than the unguis, and almost as long as the unguis, whereas most other species in the $P$. obscurus species group have the accessory tooth less stout than the unguis and half the length of the unguis or less, or are without accessory tooth.

The characteristic gape in the chela of the major second pereiopod in $P$. incertus (fig. 16A, B) is also present in the new species. Periclimenes incertus is the only species in the P. obscurus species group associated with sponges. The original description by Borradaile (1915) is brief. Kemp (1922) described Periclimens impar based on a single ovigerous female collected from a sponge at Port Blair, Andaman Islands. Holthuis (1952) showed that the difference used by Kemp to separate $P$. impar from $P$. incertus is not correct and suggested that $P$. impar might by a junior synonym of $P$. incertus. Kemp (1922) separated $P$. incertus from $P$. impar by the fact that in P. impar at least one tooth of the dorsal series of the rostrum is placed on the carapace, while in $P$. incertus all teeth are situated on the rostrum proper. This does not hold as the type specimen figured by Borradaile (1917: pl. 53, fig. 7) shows a distinct tooth on the carapace. Holthuis (1959) was able to confirm this when he reexamined the type material of $P$. incertus in the collection of the University Museum of Zoology in Cambridge and synonymized $P$. impar with $P$. incertus. Specimens collected from a sponge host at Semporna, Sabah, Malaysia (figs. 8C, D, 15-17) and identified as $P$. incertus on the bases of the description by Kemp of $P$. impar were used for morphological and molecular comparisons. The specimens (fig. 8C, D) fit the colour description provided by Kemp (1922: 149): 'The specimen was found on a sponge of pinkish colour and was transparent when alive with reddish patches on the abdominal pleura' and Bruce (1980:13): 'Highly transparent, with fine paired red striae along the lower branchiostegite and abdomen.'

The new species differs morphologically from $P$. incertus (figs. 15-17) in: 1) having the ventral margin of the rostrum slightly convex (fig. $10 \mathrm{~B}$ ) while it is straight in $P$. incertus (Fig. 15B); 2) in processing one subdistal tooth on the ventral lamina on the rostrum (fig. 1oB) whereas $P$. incertus has one or two teeth there (fig. ${ }_{15} \mathrm{~B}$ ); 3 ) in having the proximalmost tooth on the rostrum situated at the level of the orbit (fig. 10A), whereas in P. incertus it is demarcated from the dorsal carine, and situated behind the orbit on the carapace proper, at the level of the hepatic spine (fig. 15A); 4) in having the setal ridges on the basis and ischium of the first pereiopod moderately developed with few long simple setae (figs. $10 \mathrm{G}, 11 \mathrm{C}$ ), whereas in $P$. incertus these ridges (fig. ${ }_{15} \mathrm{C}, \mathrm{E}, \mathrm{F}$ ) are more pronounced with more long simple setae; 5) in having the medial process on the coxa with few simple setae (fig. 10G), whereas in $P$. incertus this medial process (fig. $15 \mathrm{E}$ ) has apart from the simple setae a very long robust seta overreaching the basis; 6 ) in having the fingers of the chela of the first pereiopod as long as the palm (fig. $10 \mathrm{E}$ ), whereas in $P$. incertus the palm is distinctly longer than the fingers (fig. 15D); 7) in having the proximal cutting edge of the fingers of the chela of the second minor cheliped entire (fig. 12A), whereas in $P$. incertus small tubercles are present there (fig. 16D); 8) in having the fourth thoracic sternite with a medially notched median plate between the first pereiopods (fig. $10 \mathrm{G}$ ), whereas this is not notched in $P$. incertus (fig. $15 \mathrm{~F}$ ); 9) in having the accessory tooth of the dactyli of the ambulatory pereiopods as robust as the unguis and slightly falling short of the unguis in length (fig. 13B, D, F), whereas in $P$. incertus the accessory tooth is more robust than the unguis and as long as the unguis (fig. $17 \mathrm{~A}, \mathrm{~B}$ ). 


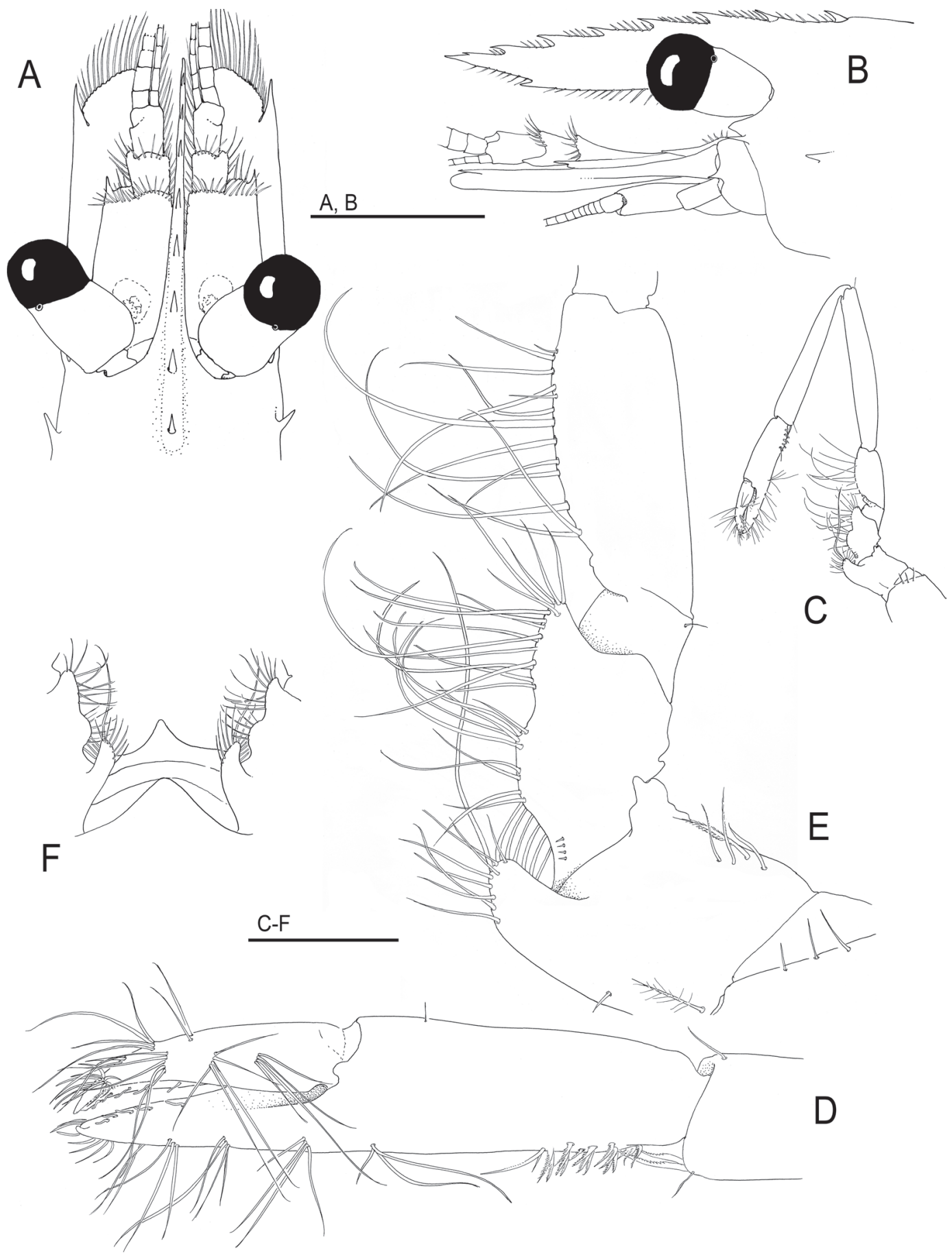

FIGURE 15 Periclimenes incertus Borradaile, 1915, ovigerous female, RMNH.CRUS.D.53946. A, rostrum and anterior appendages, dorsal view; B, idem, lateral view; C, left first pereiopod; D, idem, chela; E, idem, proximal segments; F, fourth thoracic sternites and proximal segments of first pereiopods. Scale bar: $\mathrm{A}-\mathrm{C}=1 \mathrm{~mm} ; \mathrm{D}-\mathrm{E}=0.2 \mathrm{~mm} ; \mathrm{F}=0.4 \mathrm{~mm}$. 


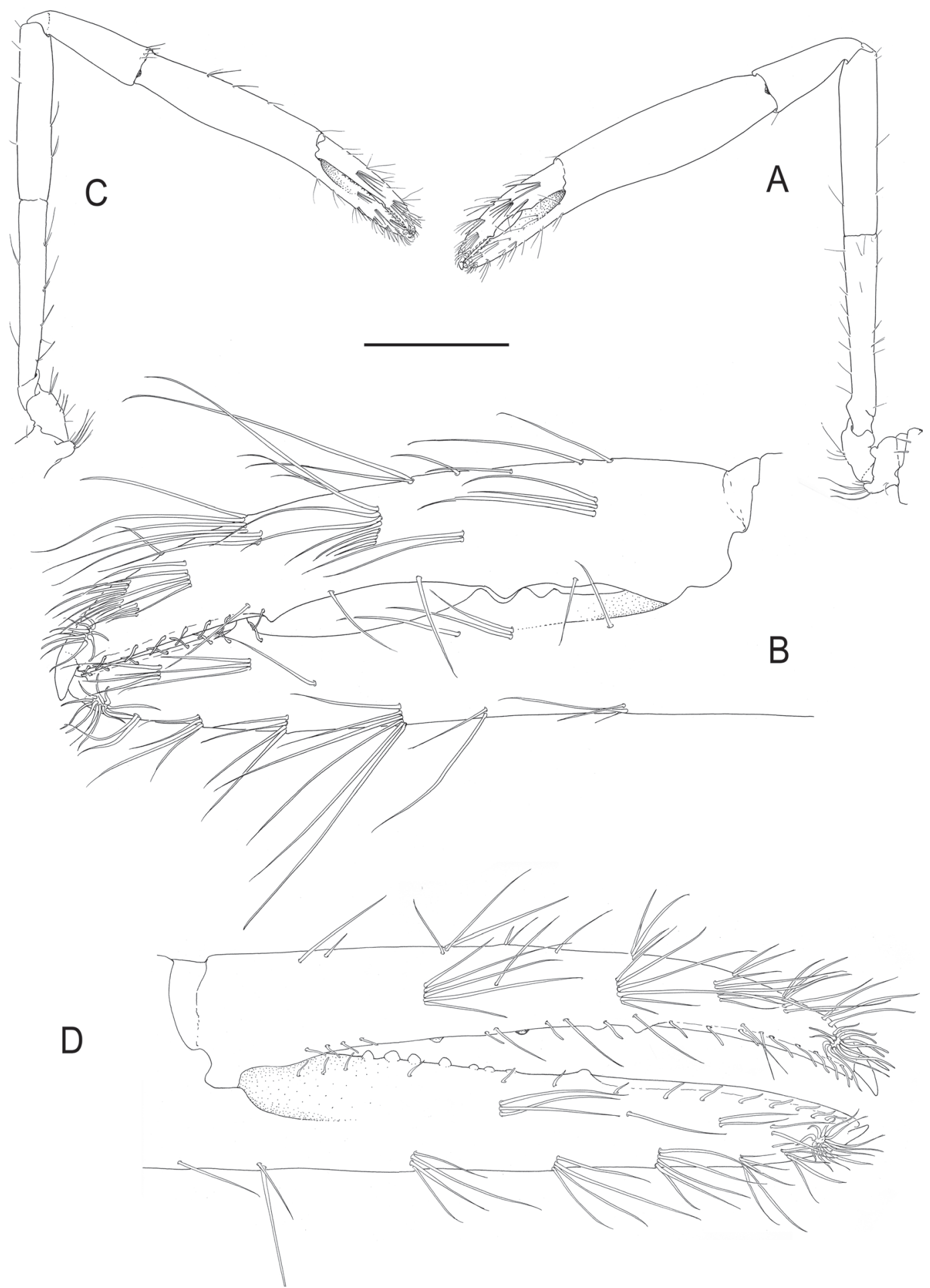

FIGURE 16 Periclimenes incertus Borradaile, 1915, ovigerous female, RMNH.CRUS.D.53946. A, left major second pereiopod; B, idem, chela; C, right minor second pereiopod; D, idem, chela. Scale bar: A, C = $1 \mathrm{~mm}$; $\mathrm{B}, \mathrm{D}=0.2 \mathrm{~mm}$. 


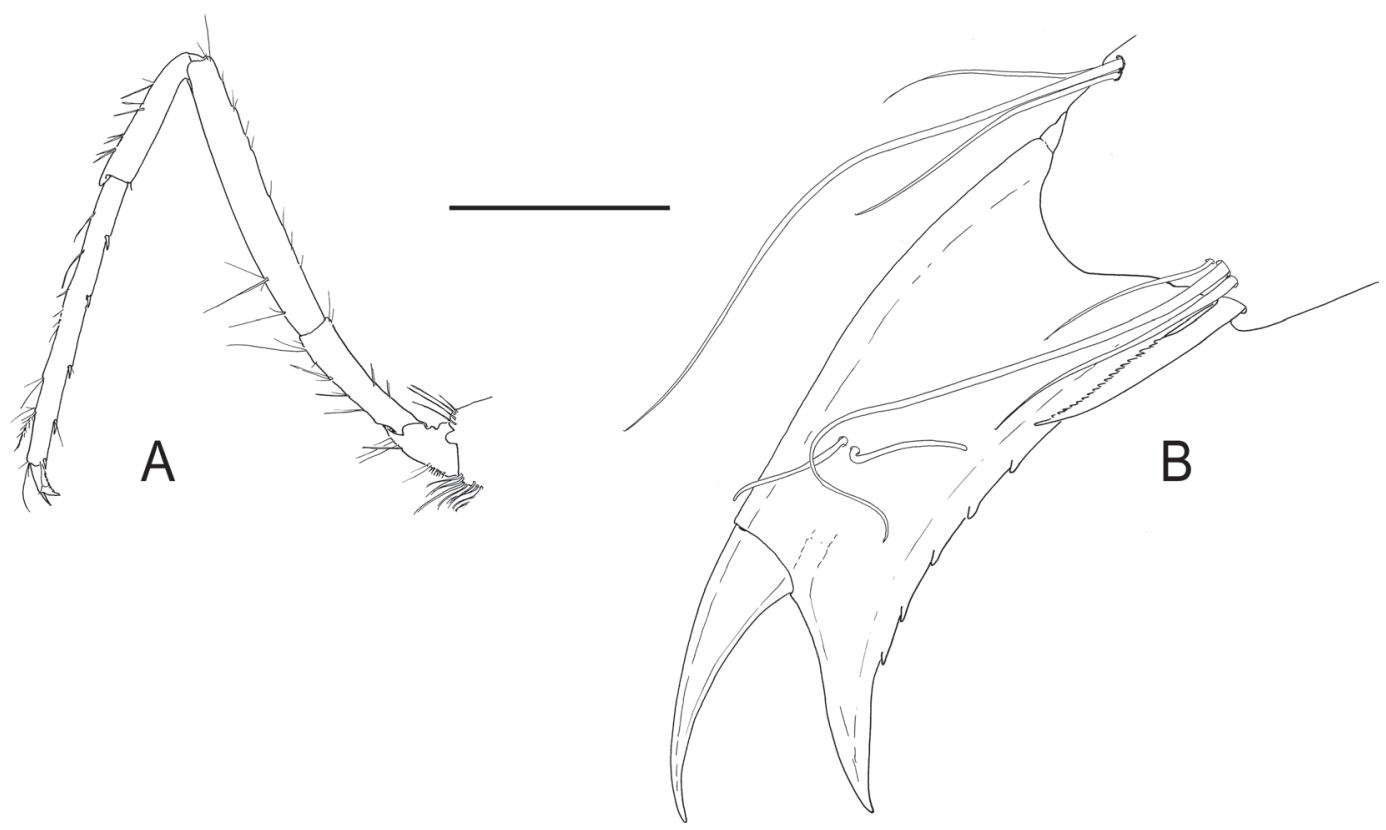

FIGURE 17 Periclimenes incertus Borradaile, 1915, ovigerous female, RMNH.CRUS.D.53946. A, left third pereiopod; B, idem dactylus. Scale bar: $\mathrm{A}=1 \mathrm{~mm} ; \mathrm{B}=0.2 \mathrm{~mm}$.

The other closely related species is the black-coral-associated Periclimenes zevinae Duriš, 1990. P. zevinae was described on the basis of a single female from the Maldives. As Duriš (1990) suspects, the specimen is not an adult, which makes comparison with adult features of the other members of the species group difficult. However, this species is similar to the new species in many respects. With $P$. incertus and the new species it shares the ventrally denticulate ambulatory dactyli, a unique character within the P. obscurus group, as well as the robust accessory tooth of the ambulatory pereiopods. P. zevinae however, has the tips of the fingers of the chela of the first pereiopods flattened, 'nail-like'. $P$. zevinae is the only species in the $P$. obscurus group with this character. $P$. subcorallum sp. nov. has the fingers not flattened, nor 'nail-like'.

Fransen (1994) recorded specimens from the Seychelles with ventrally denticulate ambulatory dactyli which he thought to be possibly conspecific with P. zevinae Ďuriš, 1990 as these specimens possess the 'nail-like' tips on the fingers of the first pereiopods. These specimens are full grown and show some differences in the dentition of the rostrum and ornamentation of the chela of the second pereiopods compared with the subadult holotype of $P$. zevinae. With regards to the second pereiopods these specimens show the same features as the P. subcorallum sp. nov. The rostrum however differs as an epigastal tooth is present which was not observed in P. subcorallum sp. nov.

\section{Discussion}

Three shrimp species of Periclimenes were found to live in association with mushroom corals of which one is new to science. Hoeksema et al. (2012) recorded 40 associations between mushroom corals and shrimp. This 
study adds another 15 different pairs of shrimp and host coral species.

Although various species of scleractinians have been recorded as hosts for palaemonid shrimps, most mushroom coral species (about $80 \%$ ) are different from other scleractinians because they are initially attached (Hoeksema \& Yeemin, 2012) but free-living when they are full-grown (Hoeksema, 1989; Gittenberger et al., 2011; Benzoni et al., 2012). This implies that they can offer space to associated fauna underneath the corals, as seen in some gastropods (Gittenberger \& Hoeksema, 2013), sessile ctenophores (Hoeksema et al., 2013b; Alamaru et al., 2016), brittle stars (Bos \& Hoeksema, 2017), and shrimps (Hoeksema \& Fransen, 2011; present study). Even large corallivorous snails can use the space underneath mushroom corals to hide themselves during daylight (Hoeksema et al., 2013a) and use this same space also to lay their eggs (Scott et al., 2017).

It is obvious that mushroom coral species with a large maximum size offer more shelter space than smaller species, while they usually also get a more convex growth form with additional room underneath (Hoeksema \& Moka, 1989; Hoeksema, 1991, 1993). Among the host species recorded, none belong to the mushroom coral genus Cycloseris, which are usually relatively small with a smooth or flat underside or encrusting, offering little shelter space (Benzoni et al., 2012; Hoeksema, 2014). Corals of free-living Cycloseris species usually occur on soft substrates causing the risk of burial (Schuhmacher, 1977; Bongaerts et al., 2012), they may be able to fragment themselves (Hoeksema \& Waheed, 2011, 2012) and move around (Hoeksema et al., 2012, 2018, 2019a, b), which is not a stable environment for shrimps and other animals that need to hide themselves underneath (Gittenberger \& Hoeksema, 2013).

It is noteworthy that five small shrimp species of the genus Periclimenes show much overlap in their host choice (table 3 ) and that these hosts belong to various coral genera that form a polyphyletic group within the family Fungiidae (Gittenberger et al., 2011). This indicates that host specificity among these shrimp species is predominantly determined at a high taxonomic level, as also seen in some other crustaceans, like gall crabs (Van der Meij et al., 2015) and copepods (Ivanenko et al., 2018).

\section{Acknowledgements}

The Marine Biodiversity Workshop was organized by Universitas Sam Ratulangi (UNSRAT in Manado) and the Bitung field station of the Research Centre of Oceanography (PPO-LIPI) in cooperation with Naturalis Biodiversity Center. Prof. Dr. Suharsono, former director of the Research Centre for Oceanography (PPO-LIPI), is acknowledged for his support. Prof. Dr. Markus T. Lasut (Universitas Sam Ratulangi) and Ir. Yosephine Tuti (PPO-LIPI) acted as workshop coordinators in cooperation with the second author. Fieldwork of the first author was funded by the Jan Joost ter Pelkwijkfonds, the L.B. Holthuis Fonds, Mej. A.M. Buitendijk Fonds and LUF International Student Fund (LISF). We thank Bastian T. Reijnen and Dick Groenenberg for their help with the molecular analyses. Zdenek Ďuriš and two anonymous reviewers are thanked for their constructive comments which considerably improved the manuscript.

\section{References}

Alamaru, A., Brokovich, E. \& Loya, Y. (2016) Four new species and three new records of benthic ctenophores (Family: Coeloplanidae) from the Red Sea. Mar. Biodivers., 46, 261-279.

Balss, H. (1914) Über einige Pontoniiden. Zool. Anz., $45,83-88$.

Downloaded from Brill.com04/26/2023 10:31:41AM 
Barnard, K.H. (1958) Further additions to the crustacean fauna-list of Portuguese East Africa. Mem. Museu Dr. Alvaro de Castro, 4, 3-23.

Benzoni, F., Arrigoni, R., Stefani, F., Reijnen, B.T., Montano, S. \& Hoeksema, B.W. (2012) Phylogenetic position and taxonomy of Cycloseris explanulata and C. wellsi (Scleractinia: Fungiidae): lost mushroom corals find their way home. Contrib. Zool., 81, 125-146.

Berggren, M. (1994) Periclimenes nomadophila and Tuleariocaris sarec, two new species of pontoniine shrimps (Decapoda: Pontoniinae), from Inhaca Island, Moçambique. J. Crustacean Biol., 14, 782-802.

Bongaerts, P., Hoeksema, B.W., Hay, K.B. \& HoeghGuldberg, O. (2012) Mushroom corals overcome live burial through pulsed inflation. Coral Reefs, 31, 399 .

Borradaile, L.A. (1915) Notes on Carides. Ann. Mag. Nat. Hist., 8, 205-213.

Borradaile, L.A. (1917) The Percy Sladen Trust Expedition to the Indian Ocean in 1905, under the leadership of Mr. J. Stanley Gardiner, M.A. No. VIII. On the Pontoniinae. Trans. Linn. Soc. London, Ser. 2, Zool., 17, 323-396.

Bos, A.R. \& Hoeksema, B.W. (2017) Mushroom corals (Fungiidae) in the Davao Gulf, Philippines, with records of associated fish and other cryptofauna. Raffles Bull. Zool., 65, 198-206.

Brinkmann, B.W. \& Fransen, C.H.J.M. (2016) Identification of a new stony coral host for the anemone shrimp Periclimenes rathbunae Schmitt, 1924 with notes on the host-use pattern. Contrib. Zool., 85, 437-456.

Bruce, A.J. (1966) Note on some Indo-Pacific Pontoniinae. XI. A re-examination of Philarius lophos Barnard, with the designation of a new genus, Ischnopontonia. Bull. Mar. Sci., 16, 584-598.

Bruce, A.J. (1967) Notes on some Indo-Pacific Pontoniinae III-IX. Description of some new genera and species from the western Indian Ocean and the South China Sea. Zool. Verh. Leiden, 87, $1-73$.

Bruce, A.J. (1969) Preliminary descriptions of sixteen new species of of the genus Peri- climenes Costa, 1844 (Crustacea, Decapoda, Natantia, Pontoniinae). Zool. Meded. Leiden, 43, 253-278.

Bruce, A.J. (1970) Notes on some Indo-Pacific Pontoniinae. XV. Hamopontonia corallicola gen. nov., sp. nov. a new pontoniid shrimp from Hong Kong. Crustaceana, 18, 37-48.

Bruce, A.J. (1973a) Notes on some Indo-Pacific Pontoniinae. XXII. Pliopontonia furtiva gen. nov., sp. nov., a new shrimp associated with a corallimorph zoantharian. Crustaceana, 24, 97-109.

Bruce, A.J. (1973b) Notes on some Indo-Pacific Pontoniinae. XXIII. Tectopontonia maziwiae gen. nov., sp. nov., a new coral associate from Tangaynika (Decapoda, Palaemonidae). Crustaceana, 24, 169-180.

Bruce, A.J. (1976a) A report on a small collection of shrimps from the Kenya National Marine Parks at Malindi, with notes on selected species. Zool. Verh. Leiden, 145, 1-72.

Bruce, A.J. (1976b) A report on some pontoniid shrimps collected from the Seychelle Islands by the F.R.V. Manihine, 1972, with a review of the Seychelles pontoniid shrimp fauna. Zool.J. Linn. Soc., 59, 89-153.

Bruce, A.J. (1979) Ctenopontonia cyphastreophila, a new genus and species of coral associated pontoniine shrimp from Eniwetok Atoll. Bull. Mar. Sci., 29, 423-435.

Bruce, A.J. (1980) On some pontoniine shrimps from Noumea, New Caledonia. Cah. l'Indo-Pac., 2, 1-39.

Bruce, A.J. (1989) Periclimenes gonioporae sp. nov. (Crustacea: Decapoda: Palaemonidae), a new coelenterate-associated shrimp. Beagle Rec. Mus. Art Gall. North. Territ., 6, 149-156.

Bruce, A.J. (1997) A new pontoniine shrimp genus (Crustacea: Decapoda) from the Yemen, with a note on other species. J. Nat. Hist., 31, 1213-1222.

Bruce, A.J. (1998) Pontoniine shrimps from Moreton Bay, Queensland (Crustacea: Decapoda, Pontoniinae). Mem. Queensl. Mus., 42, 387-398. Bruce, A.J. (2007) Palaemonoid shrimps from the Dampier Archipelago (Crustacea: Decapoda), Downloaded from Brill.com04/26/2023 10:31:41AM 
with a review of the Western Australian pontoniine shrimp fauna. Rec. West. Aust. Mus., 73 (Suppl.), 97-129.

Bruce, A.J. (2010) Pontoniine shrimps (Crustacea: Decapoda: Palaemonidae) from the CReefs 2009 Heron Island Expedition, with a review of the Heron island pontoniine fauna. Zootaxa, 2541, 50-68.

Bruce, A.J. \& Coombes, K.E. (1995) The palaemonoid shrimp fauna (Crustacea: Decapoda: Caridea) of the Cobourg Peninsula, Northern Territory. Beagle Rec. Mus. Art Gall. North. Territ., 12, 101-144.

Clark, A.H. (1919) Some necessary changes in crustacean nomenclature. Proc. Biol. Soc. Wash., 32, 199.

Costa, O.G. (1844) Su due nuovi generi di Crostacei decapodi macrouri. Ann. Accad. Aspir. Nat. Napoli, 2, 285-292.

Dana, J.D. (1846) United States Exploring Expedition during the years 1838-1842. Zoophytes, 7, 1-740. Lea and Blanchard, Philadelphia.

Dana, J.D. (1852) Conspectus Crustaceorum, quae in Orbis Terrarum circumnavigatione, Carolo Wilkese Classe Republicae Foederatae Duce, lexit et descripsit. Proc. Acad. Nat. Sci. Phil., $1852,10-28$.

De Grave, S. (1998) Pontoniinae (Decapoda, Caridea) associated with Heliofungia actiniformis (Scleractinia) from Hansa Bay, Papua New Guinea. Belg. J. Zool., 128, 13-22.

De Grave, S. (2000) Caridean shrimps (Crustacea, Decapoda) from Hansa Bay, Papua New Guinea: Palaemonidae and Gnathophyllidae. Bull. K. Belg. Inst. Natuurw. Biol., 70, 119-148.

De Grave, S. \& Fransen, C.H.J.M. (2011) Carideorum catalogus: the Recent species of the dendrobranchiate, stenopodidean, procarididean and caridean shrimps (Crustacea: Decapoda). Zool. Meded. Leiden, 85, 195-589.

Duriš, Z. (1990) Two new species of the palaemonid shrimp genus Periclimenes from the Maldive waters (Crustacea, Decapoda, Palaemonidae). Acta Soc. Zool. Bohemoslovacae, 54, 1-8.
Ďuriš, Z. \& Lin, C.W. (2016) The 'scorpion shrimp', a new species of the genus Metapontonia (Crustacea: Decapoda: Palaemonidae) from Taiwan, with new generic record from Papua New Guinea. Zootaxa, 4138, 474-49o.

Eilbracht J. \& Fransen, C.H.J.M. (2015) Periclimenes macrorhynchia sp. nov., a new hydrozoan-associated pontoniine shrimp (Crustacea, Decapoda, Palaemonidae) from North East Kalimantan, Indonesia. Zootaxa, 3994, 377-395.

Esper, E.J.C. (1797) Fortsetzungen der Pflanzenthiere in Abbildungennach der Natur mit Farben erleuchtet nebst Beschreibungen. Erster Theil. Nürnberg, pp. 1-230.

Folmer, O., Black, M., Hoeh,W., Lutz, R. \& Vrijenhoek, R. (1994) DNA primers for amplification of mitochondrial cytochrome $c$ oxidase subunit I from diverse metazoan invertebrates. Mol. Mar. Biol. Biotechnol., 3, 294-299.

Fransen, C.H.J.M. (1989) Notes on caridean shrimps collected during the Snellius-II Expedition. I. Associates of Anthozoa. Neth. J. Sea Res., 23, 131-147.

Fransen, C.H.J.M. (1994) Marine palaemonoid shrimps of the Netherlands Seychelles Expedition 1992-1993. Zool. Verh. Leiden, 297, 85-152.

Fransen, C.H.J.M. (1997) Indonesian pontoniine shrimps. In: T. Tomascik, A.J. Mah, A. Nontji \& M. Kasim Moosa (Eds), The Ecology of the Indonesian Seas, Part II, Box 21.3, pp. 1064-1075.

Fransen, C.H.J.M. \& Holthuis, L.B. (2007) Vir smiti spec. nov., a new scleractinian associated pontoniine shrimp (Crustacea: Decapoda: Palaemonidae) from the Indo-West Pacific. Zool. Meded. Leiden, 81, 101-114.

Fransen, C.H.J.M. \& Rauch, C. (2013) Hamodactylus macrophthalmus spec. nov., a new coral-associated pontoniine shrimp (Decapoda, Caridea, Palaemonidae) from Indonesia. Zootaxa, 3635, 286-296.

Gittenberger, A. \& Hoeksema, B.W. (2013) Habitat preferences of coral associated wentletrap snails (Gastropoda: Epitoniidae). Contrib. Zool., $82,1-25$. 
Gittenberger, A., Reijnen, B.T. \& Hoeksema, B.W. (2011) A molecularly based phylogeny reconstruction of mushroom corals (Scleractinia: Fungiidae) with taxonomic consequences and evolutionary implications for life history traits. Contrib. Zool., 80, 107-132.

Hall, T.A. (2001) Bioedit: A User-Friendly Biological Sequence Alignment Editor and Analysis, version 5.09. Department of Microbiology, North Carolina State University, North Carolina.

Hoeksema, B.W. (1989) Taxonomy, phylogeny and biogeography of mushroom corals (Scleractinia: Fungiidae). Zool. Verh. Leiden, 254, 1-295.

Hoeksema, B.W. (1991) Evolution of body size in mushroom corals (Scleractinia: Fungiidae) and its ecomorphological consequences. Neth. J. Zool., 41, 122-139.

Hoeksema, B.W. (1993) Phenotypic corallum variability in Recent mobile reef corals. Cour. Forsch.-Inst. Senckenb., 164, 263-272.

Hoeksema, B.W. (2012) Evolutionary trends in onshore-offshore distribution patterns of mushroom coral species (Scleractinia: Fungiidae). Contrib. Zool., 81, 199-221.

Hoeksema, B.W. (2014) The "Fungia patella group" (Scleractinia, Fungiidae) revisited with a description of the mini mushroom coral Cycloseris boschmai sp. n. ZooKeys, 371, 57-84.

Hoeksema, B.W. (2017) The hidden biodiversity of tropical coral reefs. Biodiversity, 18, 8-12.

Hoeksema, B.W. \& Fransen, C.H.J.M. (2011) Space partitioning by symbiotic shrimp species cohabitating in the mushroom coral Heliofungia actiniformis at Semporna, eastern Sabah. Coral Reefs, 30, 519 .

Hoeksema, B.W. \& Moka, W. (1989) Species assemblages and ecomorph variation of mushroom corals (Scleractinia: Fungiidae) related to reef habitats in the Flores Sea. Neth. J. Sea Res., 23, 149-16o.

Hoeksema, B.W. \& Waheed, Z. (2011) Initial phase of autotomy in fragmenting Cycloseris corals at Semporna, eastern Sabah, Malaysia. Coral Reefs, 30, 1087 .
Hoeksema, B.W. \& Waheed, Z. (2012) Onset of autotomy in an attached Cycloseris coral. Galaxea J. Coral Reef Stud., 14, 1-2.

Hoeksema, B.W. \& Yeemin, T. (2011) Late detachment conceals serial budding by the free-living coral Fungia fungites in the Inner Gulf of Thailand. Coral Reefs, 30, 975.

Hoeksema, B.W., van der Meij, S.E.T. \& Fransen, C.H.J.M. (2012) The mushroom coral as a habitat. J. Mar. Biol, Assoc. U.K., 92, 647-663.

Hoeksema, B.W., Scott, C. \& True, J.D. (2013a) Dietary shift in corallivorous Drupella snails following a major bleaching event at Koh Tao, Gulf of Thailand. Coral Reefs, 32, 423-428.

Hoeksema, B.W., Waheed, Z. \& Alamaru, A. (2013b) Out of sight: aggregations of epizoic comb jellies underneath mushroom corals. Coral Reefs, 32,1065 .

Hoeksema, B.W., Bouwmeester, J., Range, P. \& BenHamadou, R. (2018) A large aggregation of selffragmenting mushroom corals in the Arabian / Persian Gulf. Ecology, 99, 1236-1238.

Hoeksema, B.W., Sellanes, J. \& Easton, E.E. (2019a) A high-latitude, mesophotic Cycloseris field at $85 \mathrm{~m}$ depth off Rapa Nui (Easter Island). Bull. Mar. Sci., 95, 101-102.

Hoeksema, B.W., Giyanto \& Suharsono (2019b) The role of maximum shelf depth versus distance from shore in explaining a diversity gradient of mushroom corals (Fungiidae) off Jakarta. Diversity, $11,46$.

Holthuis, L.B. (1951) A general revision of the Palaemonidae (Crustacea, Decapoda Natantia) of the Americas. I. The subfamilies Euryrhynchinae and Pontoniinae. Occas. Pap. Allan Hancock Found., 11, 1-332.

Holthuis, L.B. (1952) The Decapoda of the Siboga Expedition. Part XI. The Palaemonidae collected by the Siboga and Snellius Expeditions with remarks on other species. II. Subfamily Pontoniinae. Siboga Exped. Monogr., 39a 10, 1-252.

Holthuis, L.B. (1959) Results of the reexamination of the type specimens of some species belonging to the subfamilies Pontoniinae and 
Palaemoninae (Crustacea Decapoda Macrura). Zool. Meded. Leiden, 36, 193-200.

Horká, I., De Grave, S., Fransen, C.H.J.M., Petrusek, A. \& Duriš, Z. (2016) Multiple host switching events shape the evolution of symbiotic palaemonid shrimps (Crustacea: Decapoda). Sci. Rep., 6, 26486.

Ivanenko, V.N., Hoeksema, B.W., Mudrova, S.V., Nikitin, M.A., Martínez, A., Rimskaya-Korsakova, N.N., Berumen, M.L. \& Fontaneto, D. (2018) Lack of host specificity of copepod crustaceans associated with mushroom corals in the Red Sea. Mol. Phylogenet. Evol., 127, 770-780.

Jukes, T.H. \& Cantor, C.R. (1969) Evolution of Protein Molecules. Academic Press, New York, pp. 21-132.

Kemp, S. (1922) Notes on Crustacea Decapoda in the Indian Museum, 15. Pontoniinae. Rec. Ind. Mus., 24, 113-228.

Klunzinger, C.B. (1879) Die Korallenthiere des Rothen Meeres, 3. Theil: Die Steinkorallen. Zweiter Abschnitt: Die Asteraeaceen und Fungiaceen. Gutmann, Berlin, pp. 1-100.

Lamarck, J.B. (1801) Système des animaux sans vertèbres, ou tableau général des classes, des ordres et des genres de ces animaux; Présentant leurs caractères essentiels et leur distribution, d'apres la considération de leurs rapports naturels et de leur organisation, et suivant l'arrangementétabli dans les galeries du Muséum d'Histoire Naturelle, parmi leurs dépouilles conservées; Précédé du discours d'ouverture du Cours de Zoologie, donné dans le Muséum National d'Histoire Naturelle l'an 8 de la République. Published by the author and Deterville, Paris, viii $+432 \mathrm{pp}$.

Linnaeus, C. (1758) Systema Naturae per regna tria naturae, secundum classes, ordines, genera, species, cum characteribus, differentiis, synonymis, locis. Editio decima, reformata. Laurentius Salvius, Holmiae, ii, 824 pp.

Meij, S.E.T. van der, Fransen, C.H.J.M., Pasman, L.R. \& Hoeksema, B.W. (2015) Phylogenetic ecology of gall crabs (Cryptochiridae) as associates of mushroom corals (Fungiidae). Ecol. Evol., 5, 5770-5780.
Nemenzo, F. (1955) Systematic studies on Philippine shallow water scleractinians: I. Suborder Fungiida. Nat. Appl. Sci. Bull. Univ. Philipp., 15, 3-84.

Okuno, J. (1999) Izocaris masudai, new genus, new species (Decapoda: Caridea: Palaemonidae), a sea anemone associate from Japan. J. Crustacean Biol., 19, 397-407.

Okuno, J. (2009) Pliopontonia harazakii sp. nov., a new species of coral-associated shrimp (Decapoda: Caridea: Palaemonidae) from Yaku-shima Island, southern Japan. Bulletin of the National Museum of Nature and Science (A, Zoology), Suppl., 3, 105-113.

Okuno, J. \& Bruce, A.J. (2010) Designation of Ancylomenes gen. nov., for the 'Periclimenes aesopius species group'(Crustacea: Decapoda: Palaemonidae), with the description of a new species and a checklist of congeneric species. In: S. De Grave \& C.H.J.M. Fransen (Eds), Contributions to shrimp taxonomy. Zootaxa, 2372, 85-105.

Pallas, P.S. (1766) Elenchus zoophytorum sistens generum adumbrationes generaliores et specierum cognitarum succintas descriptiones, cum selectis auctorum synonymis. Fransiscum Varrentrapp, Hagae, $45^{1} \mathrm{pp}$.

Posada, D. (2008) jModelTest: Phylogenetic model averaging. Mol. Biol. Evol., 25, 1253-1256.

Quelch, J.J. (1886) Report on the Reef-corals collected by H.M.S. 'Challenger' during the years 1873-76. Report on the Scientific Results of the Voyage of H.M.S. Challenger during the years 1873-1876. Zoology, 16 (part 46), 1-203.

Quoy, J.R.C. \& Gaimard, J.P. (1833) Zoologie IV: Zoophytes.In:Zoologie.Voyagedelacorvettel'Astrolabe : exécuté par ordre du roi, pendant les années 1826-1827-1828-1829 / sous le commandement de J. Dumont d'Urville. J. Tastu, Paris, pp. 1-390.

Rafinesque, C.S. (1815) Analyse de la Nature ou Tableau de l'Univers et des corps organisés. Palerme, pp. 1-224.

Schenkel, E. (1902) Beitrag zur Kenntnis der Dekapodenfauna von Celebes. Verhandlungen der Naturforschenden Gesellschaft in Basel, 13, $485^{-585}$. 
Schuhmacher, H. (1977) Ability in fungiid corals to overcome sedimentation. Proc. 3rd Int. Coral Reef Symp., 1, 503-509.

Scott, C.M., Mehrotra, R. \& Hoeksema, B.W. (2017) In-situ egg deposition by corallivorous snails on mushroom corals at Koh Tao (Gulf of Thailand). J. Mollusc. Stud., 83, 360-362.

Stimpson, W. (1860) Prodomus descriptionis animalius evertevratorum, quae in Expeditione ad Oceanum Pacificum Septentrionalem, a Republica Federata missa, C. Ringgold et J. Rodgers Ducibus, observavit et descripsit. Proc. Acad. Nat. Sci. Phil., 1860, 22-47.

Swofford, D.L. (2003) PAUP*. Phylogenetic Analysis Using Parsimony ( ${ }^{*}$ and Other Methods). Version 4. Sinauer Associates, Sunderland, MA.
Thompson, J.D., Higgins, D.G. \& Gibson, T.J. (1994) CLUSTAL W: improving the sensitivity of progressive multiple sequence alignment through sequence weighting, position-specific gap penalties and weight matrix choice. Nucleic Acids Res., 22, 4673-4680.

Verrill, A.E. (1864) List of the polyps and corals sent by the Museum of Comparative Zoology to other institutions in exchange, with annotations. Bull. Mus. Comp. Zool., 1, 29-6o.

RECEIVED: 12 NOVEMBER 2018 | REVISED AND ACCEPTED: 18 APRIL 2019

EDITOR: R. VONK 